\title{
Narrowed TCR repertoire and viral escape as a consequence of heterologous immunity
}

\author{
Markus Cornberg, ${ }^{1,2}$ Alex T. Chen, ${ }^{1}$ Lee A. Wilkinson, ${ }^{1}$ Michael A. Brehm, ${ }^{1}$ Sung-Kwon Kim, ${ }^{1}$ \\ Claudia Calcagno, ${ }^{3}$ Dario Ghersi, 3,4 Roberto Puzone, ${ }^{5}$ Franco Celada, ${ }^{3,4}$ \\ Raymond M. Welsh, ${ }^{1}$ and Liisa K. Selin ${ }^{1}$
}

\begin{abstract}
1Department of Pathology and Program in Immunology and Virology, University of Massachusetts Medical School (UMMS), Worcester, Massachusetts, USA.
${ }^{2}$ Department of Gastroenterology, Hepatology and Endocrinology, Medical School Hannover, Hannover, Germany. ${ }^{3}$ Department of Rheumatology, Hospital for Joint Diseases, New York, New York, USA. ${ }^{4}$ Department of Oncology, Biology and Genetics, University of Genoa, Genoa, Italy.

${ }^{5}$ Department of Clinical Epidemiology, National Institute for Cancer Research, Genoa, Italy.
\end{abstract}

\begin{abstract}
Why some virus-specific CD8 TCR repertoires are diverse and others restricted or "oligoclonal" has been unknown. We show here that oligoclonality and extreme clonal dominance can be a consequence of $T$ cell crossreactivity. Lymphocytic choriomeningitis virus (LCMV) and Pichinde virus (PV) encode $\mathrm{NP}_{205-212}$ epitopes that induce different but highly cross-reactive diverse TCR repertoires. Homologous viral challenge of immune mice only slightly skewed the repertoire and enriched for predictable TCR motifs. However, heterologous viral challenge resulted in a narrow oligoclonal repertoire with dominant clones with unpredictable TCR sequences. This shift in clonal dominance varied with the private, i.e., unique, specificity of the host's TCR repertoire and was simulated using affinity-based computer models. The skewing differences in TCR repertoire following homologous versus heterologous challenge were observed within the same private immune system in mice adoptively reconstituted with memory CD8 $\mathrm{T}$ cell pools from the same donor. Conditions driving oligoclonality resulted in an LCMV epitope escape variant in vivo resembling the natural Lassa virus sequence. Thus, $T$ cell oligoclonality, including extremes in clonal dominance, may be a consequence of heterologous immunity and lead to viral escape. This has implications for the design of peptide-based vaccines, which might unintentionally prime for skewed TCR responses to cross-reactive epitopes.
\end{abstract}

\section{Introduction}

An important feature of the immune system is a diverse repertoire of T cells (1) whose TCRs recognize antigens presented by MHC molecules (2). CD8 T cell responses to a single epitope are usually diverse and often use different variable region $\beta(V \beta)$ families, but even in the case of dominant $\mathrm{V} \beta$ usage, many different clonotypes may be present (3-6). Less diverse "oligoclonal" responses are sometimes seen against epitopes in HIV, CMV, EBV, and HCV infections (7-10). TCR diversity may help to control the pathogen and lower the possibility of immune evasion by $\mathrm{T}$ cell escape variants $(8,11)$. Why narrow oligoclonal repertoires are generated is poorly understood but might relate to the intrinsic structure of the epitope (12) or to repeated antigenic exposure (13). However, longitudinal studies in lymphocytic choriomeningitis virus- (LCMV-) and influenza virusinfected mice have revealed few changes and only modest narrowing of the TCR repertoire after homologous reinfection (14-17).

An immunologically naive host responds to infections differently than an experienced host, in part because of the activation of memory CD8 $\mathrm{T}$ cells that can cross-react between previously and newly encountered pathogens (18-20). Cross-reactive memory CD8 $\mathrm{T}$ cells can compete with the proliferation of naive CD8 T cells, even those that would normally be immunodominant in the primary immune response. This can lead to a change in epitopespecific $\mathrm{T}$ cell hierarchies and an altered immune response (21)

Nonstandard abbreviations used: CDR3, third complementarity-determining region; ICS, intracellular cytokine staining; LCMV, lymphocytic choriomeningitis virus; PV, Pichinde virus; V $\beta$, variable region of TCR $\beta$-chain.

Conflict of interest: The authors have declared that no conflict of interest exists. Citation for this article: J. Clin. Invest. 116:1443-1456 (2006). doi:10.1172/JCI27804. manifested as either protective immunity or enhanced immunopathology $(22,23)$. LCMV and Pichinde virus (PV) encode highly cross-reactive subdominant $\mathrm{K}^{\mathrm{b}}$-restricted $\mathrm{NP}_{205-212}$ epitopes sharing 6 of 8 amino acids. Heterologous virus infection of mice immune to the other virus leads to proliferation of $\mathrm{NP}_{205}$-specific CD8 T cells, resulting in a dominance for this normally subdominant epitope (21). Here we show that a surprisingly small subset of this highly cross-reactive $T$ cell population expands, modulating clonal dominance and resulting in a narrowed oligoclonal TCR repertoire, and that unpredictable clonal dominance patterns are determined by the private, i.e., unique, TCR specificities of each immune host. Viral escape may be a consequence.

\section{Results}

LCMV- or PV-induced CD8 T cells specific to $\mathrm{NP}_{205}$ show widespread functional cross-reactivity. The cross-reactive subdominant epitopes LCMV NP $205-212$ (YTVKYPNL) and PV NP $205-212$ (YTVKFPNM) differ in 2 amino acids in their MHC class I binding motifs but are similar in the positions available for TCR interaction. We questioned whether $\mathrm{NP}_{205}$-specific CD8 $\mathrm{T}$ cells induced during primary infection could recognize the heterologous $\mathrm{NP}_{205}$ peptide and mediate cross-reactive effector function, as assessed by IFN- $\gamma$ production (Figure 1). Upon LCMV infection, LCMV NP 205 peptide induced $3.0 \% \pm 1.2 \%$ (range: $1.1-4.5 \% ; n=10$ ) and the PV $\mathrm{NP}_{205}$ induced $3.1 \% \pm 1.3 \%$ (range: $0.9-4.7 \% ; n=10$ ) of the CD8 T cells to produce IFN- $\gamma$. During PV infection, the $\mathrm{PV} \mathrm{NP}_{205}$ peptide induced $0.54 \% \pm 0.33 \%$ (range: $0.19-1.1 \% ; n=12$ ), while the LCMV $\mathrm{NP}_{205}$ peptide induced $0.39 \% \pm 0.23 \%$ (range: $0.13-0.87 \% ; n=12$ ) of the CD8 T cells to produce IFN- $\gamma$. This suggests that the PV and LCMV NP 205 peptides could stimulate equivalent portions of the 

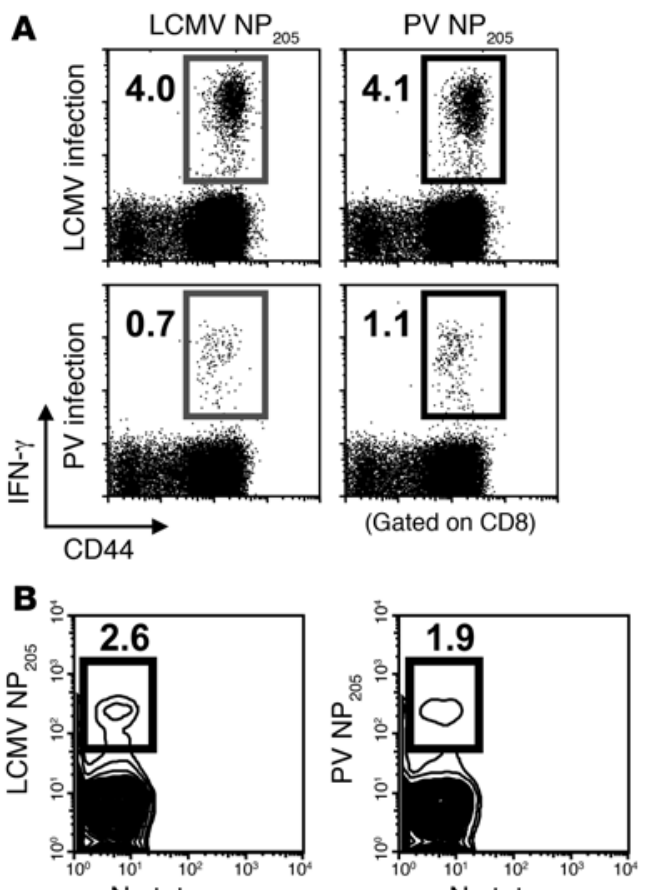

No tetramer
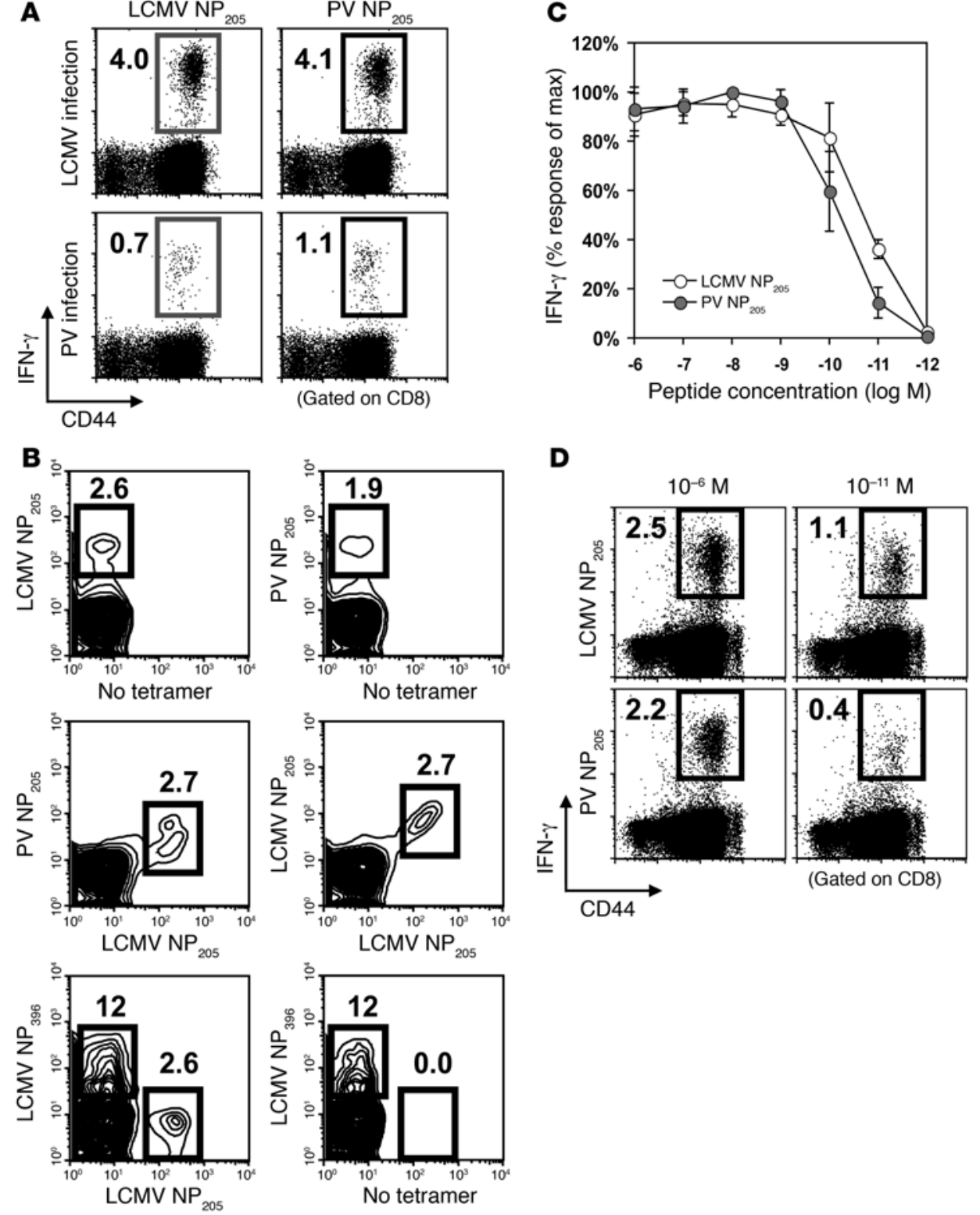

Figure 1

CD8 T cells specific to LCMV NP 205 and PV $\mathrm{NP}_{205}$ show widespread cross-reactivity at effector level. (A) Intracellular IFN- $\gamma$ assay. Splenocytes from day 8 LCMV- or PV-infected B6 mice were stimulated with the indicated peptides. Shown are gated CD8 cells stained for CD44 ( $x$ axis) and IFN- $\gamma$ ( $y$ axis). Numbers in the upper-left corners represent the percentage of CD8-producing IFN- $\gamma$. The background IFN- $\gamma$ response was less than $0.1 \%$. This is representative of 3 experiments with 2-5 mice/group. (B) Double-tetramer staining. Splenocytes were stained with anti-CD8 and the indicated tetramers. Numbers represent the percentage of gated CD8 T cells binding tetramers. This is representative of 2 experiments with 3 mice/ group. (C and D) Peptide titration. Production of IFN- $\gamma$ by splenocytes $(n=3)$ in response to different concentrations of LCMV NP 205 or $\mathrm{PV} \mathrm{NP}_{205}$ peptide. (C) IFN- $\gamma$ responses plotted as a percentage of the maximal response (\% of max). IFN- $\gamma$ responses without peptide stimulation were subtracted from each value. Differences between LCMV $\mathrm{NP}_{205}$ and PV NP 205 were statistically significant at a peptide concentration of $10^{-11} \mathrm{M}(P=0.006)$. FACS plots of data for a representative mouse of 3 with stimulation of $10^{-6}$ and $10^{-11} \mathrm{M}$ of the indicated peptide are shown in D. Numbers recorded in the upper-left corners represent the percentage of gated CD8 T cells producing IFN- $\gamma$.
LCMV-induced CD8 T cells to produce IFN- $\gamma$. The LCMV NP 205 peptide stimulated a similar but not completely equivalent portion $(76 \% \pm 21 \% ; n=12)$ of the PV-induced CD8 T cells to produce IFN- $\gamma$ compared with the $\mathrm{PV} \mathrm{NP}_{205}$ peptide (Figure $1 \mathrm{~A}$ ).

Widespread cross-reactivity was also demonstrated by staining $\mathrm{T}$ cells from day $8 \mathrm{LCMV}$-infected mice with tetramers specific to both peptides (Figure 1B). The double-tetramer staining revealed 2 discrete populations when compared with the costaining pattern using LCMV NP 205 tetramers in 2 different colors (Figure $1 \mathrm{~B}$ ). The majority of the double tetramer-positive cells were dim for the PV NP 205 tetramer as compared with the LCMV NP 205 tetramer, suggesting that the $\mathrm{NP}_{205}$ population may contain cells having differing avidities to LCMV NP 205 and $\mathrm{PV} \mathrm{NP}_{205}$, though formal estimates of affinities and avidities were not done. Correspondingly, in mice acutely infected with $\mathrm{LCMV}$, the $\mathrm{PV} \mathrm{NP_{205 }}$ peptide at lower concentrations stimulated significantly fewer cells to produce IFN- $\gamma$ than did the LCMV peptide (Figure 1, C and D).
TCR V $\beta$ repertoires of $N P_{205}$-specific CD8 $T$ cells differ in $L C M V$ and $P V$ infection. We investigated the TCR V $\beta$ repertoires by costaining lymphocytes with all available $\mathrm{V} \beta$ mAbs (V $\beta 2-14)$ in tetramer or intracellular cytokine staining (ICS) assays. Most $\mathrm{NP}_{205}$-specific $\mathrm{T}$ cells did not costain with the anti-V $\beta$ antibodies, though some stained with V $\beta 5$ (Figure $2 \mathrm{~A}$ ). To determine which $\mathrm{V} \beta$ family was contributing to the unknown V $\beta$ population, we analyzed LCMV $\mathrm{NP}_{205}$ tetramer-sorted cells by RT-PCR using specific primers for V $\beta 1$-18. In 3 LCMV-infected mice, the LCMV NP 205 -specific CD8 T cells showed a very strong amplification of the V $\beta 16$ family (Figure 2, $\mathrm{B}$ and $\mathrm{C})$. The V $\beta 16$ PCR product was subcloned and sequenced and shown to be highly diverse, with some higher-frequency clones but many low-frequency clones (Figure 2D and Supplemental Table 1; supplemental material available online with this article; doi:10.1172/ JCI27804DS1; sequencing data are shown in Supplemental Tables 1-21). In acute PV infection, sorted PV $\mathrm{NP}_{205}$-specific CD8 T cells also demonstrated a strong amplification of the V $\beta 16$ family (data 

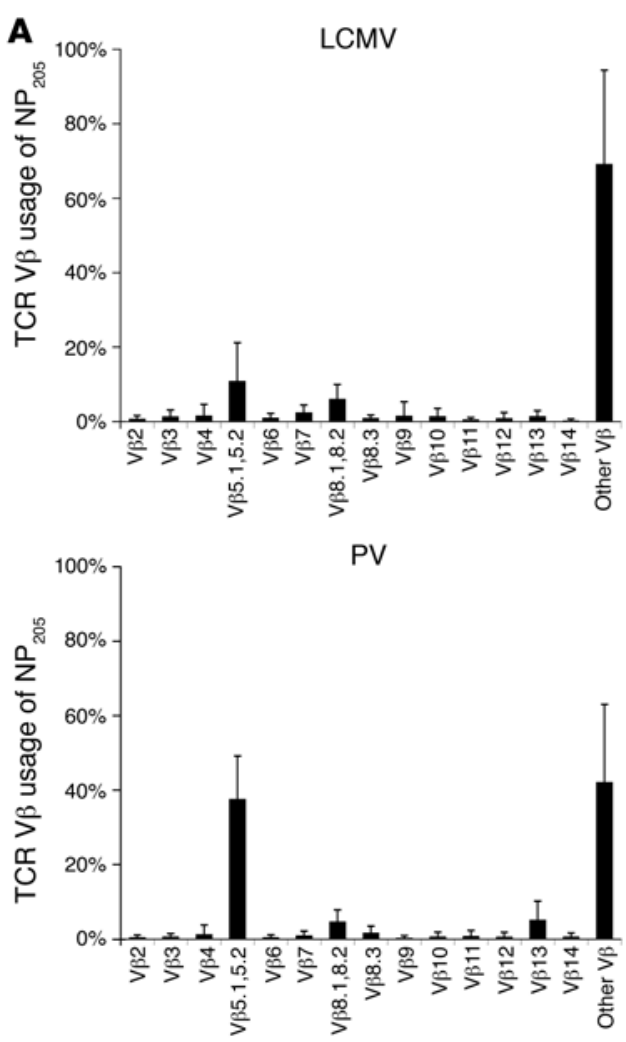

B

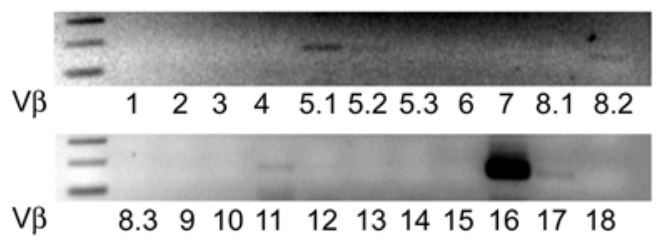

C

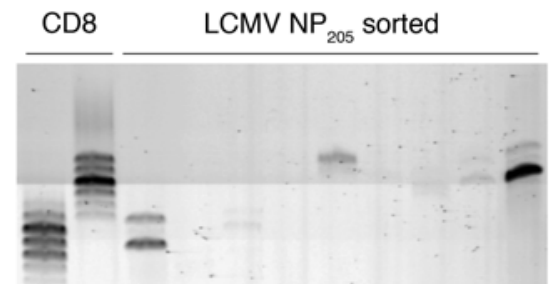

VB $\begin{array}{lllllllllll}5.1 & 16 & 5.1 & 6 & 8.2 & 8.3 & 9 & 10 & 11 & 12 & 16\end{array}$
D

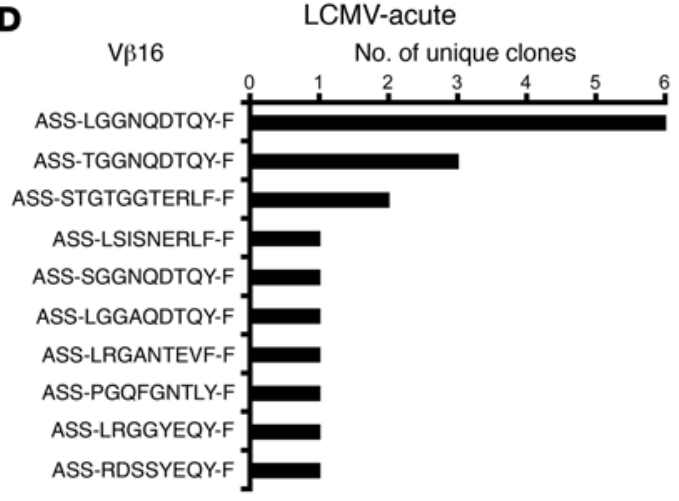

E

Vß16

PV-acute

No. of unique clones

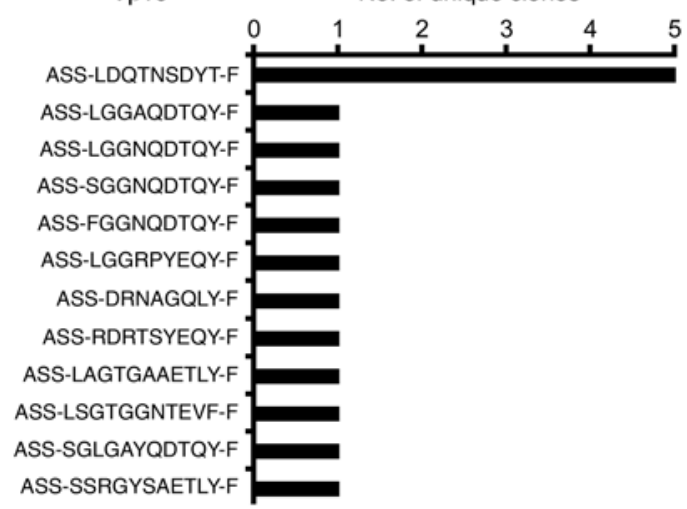

$\mathbf{F}$

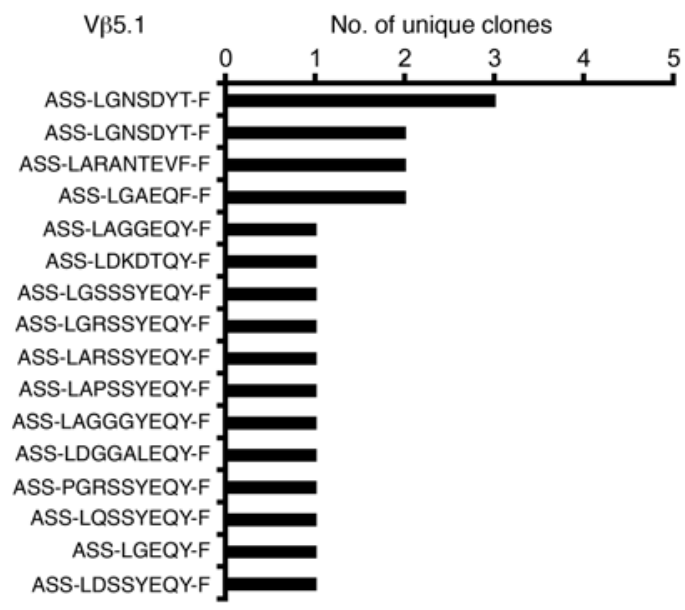

Figure 2

Differences in the TCR V $\beta$ repertoire of CD8 T cells specific to LCMV NP 205 and PV NP 205 . (A) Splenocytes from day 8 LCMV- or PVinfected mice $(n=8)$ were stimulated with LCMV NP 205 , or $\mathrm{PV} \mathrm{NP}_{205}$ peptides in an ICS assay and then stained with $\mathrm{V} \beta$-specific $\mathrm{mAbs}$. The percentage of $\mathrm{V} \beta$ usage was calculated after gating on the IFN- $\gamma$-positive CD8 T cell population. The percentage of other $\mathrm{V} \beta$ (V $\beta 15-18$ were not included in the antibody pool) was calculated by subtracting the sum of V $\beta 2-14$ from $100 \%$. (B and C) TCR V $\beta$ mRNA expression of LCMV NP 205 -specific CD8 T cells. LCMV NP 205 tetramer-positive CD8 T cells were sorted from PBMCs 8 days after LCMV infection, and RNA was isolated. RT-PCR was performed with specific primers for V $\beta 1-18$ (B). Spectratype analysis with specific primers for the indicated V $\beta$ families (C). (D) The TCR V $\beta 16$ repertoire of acute LCMV-infected mice is diverse. This shows the CDR3 amino acid sequence and the frequency of each unique $\mathrm{V} \beta 16 \mathrm{LCMV} \mathrm{NP}{ }_{205}-$ sorted CD8 T cell clone represented in $\mathbf{B}$. Clones with the same amino acid sequence that are plotted more than once have a different nucleic acid sequence. T cell clones with an XGGX-J $\beta 2.5$ (QDTQY-F) motif dominate the response. (E) The TCR V $\beta 16$ repertoire of acute PV-infected mice is diverse. (F) The TCR V $\beta 5.1$ repertoire of acute PV-infected mice is diverse. 
A

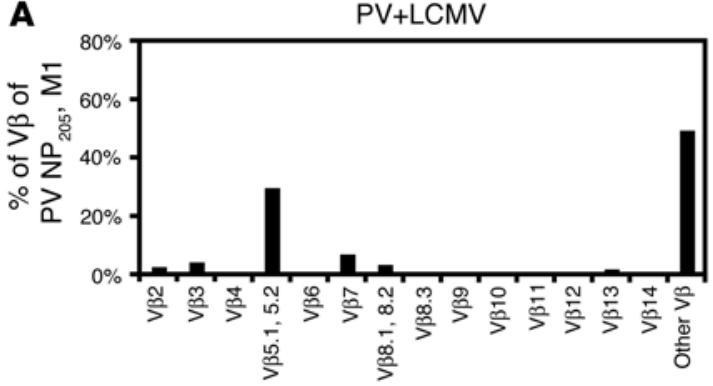

B

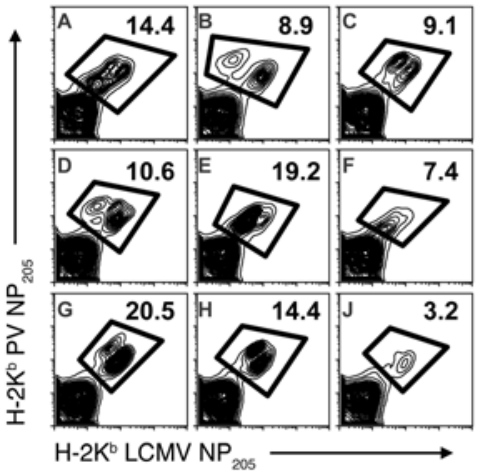

C

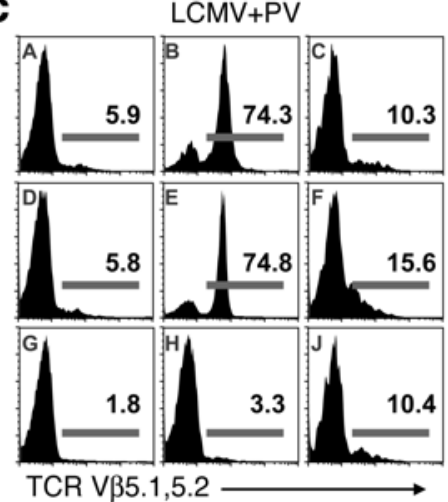

PV+LCMV

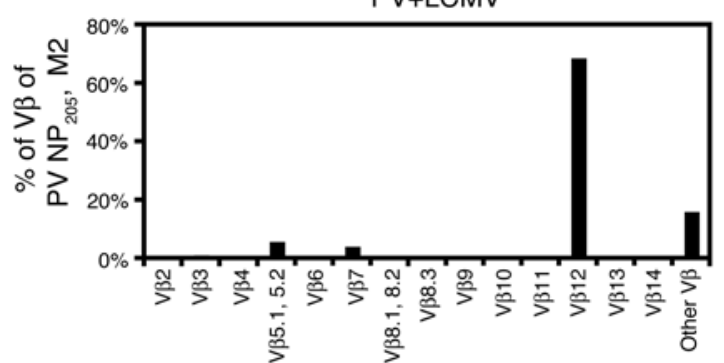

D

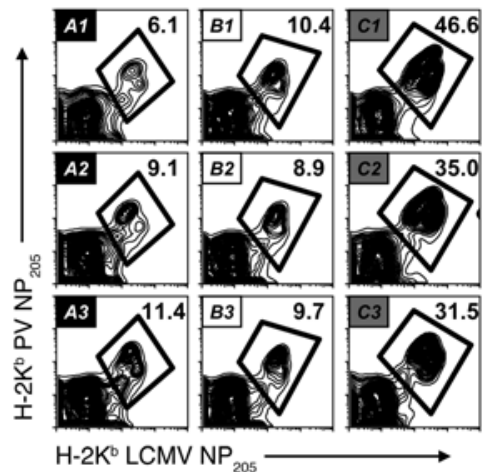

E
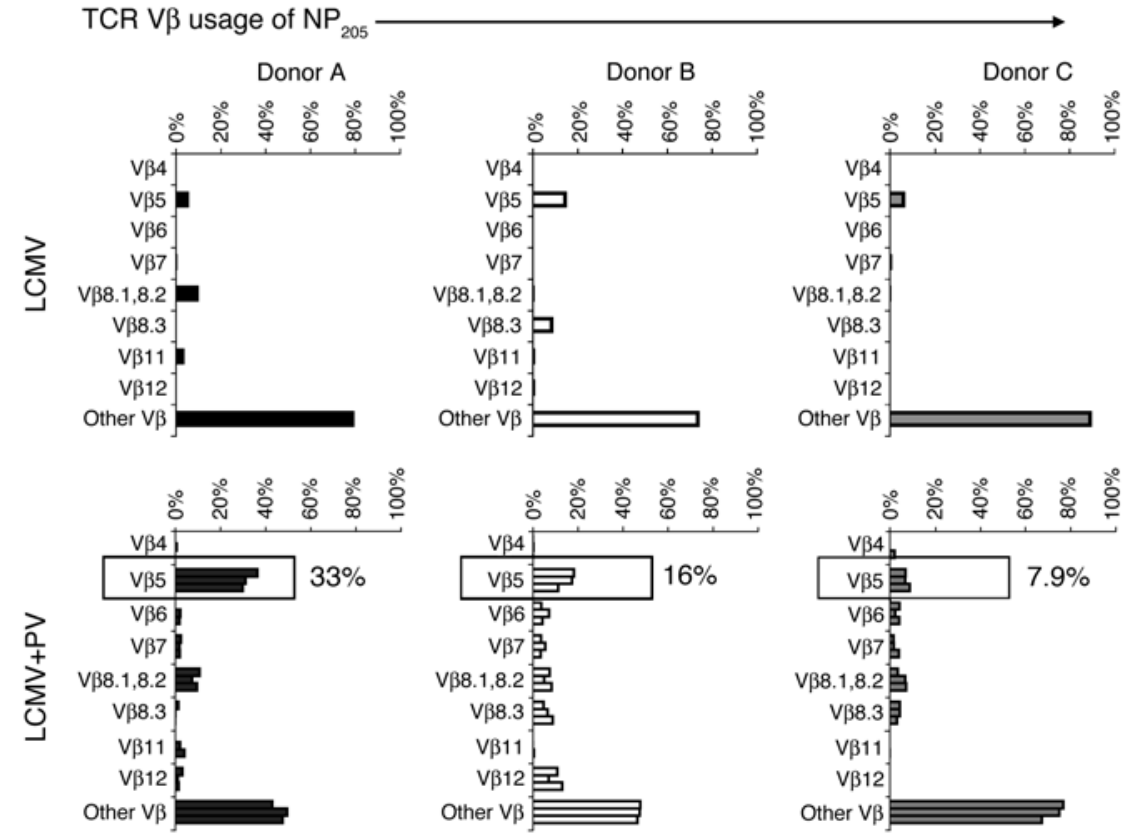

Figure 3

Variability and skewing of $\mathrm{NP}_{205}$-specific $\mathrm{T}$ cells dependent on private specificity after heterologous infection. (A) TCR V $\beta$ repertoire of PV NP 205 tetramer-positive cells of 2 PV-immune mice 8 days after LCMV challenge (PV+LCMV). (B and C) Double-tetramer staining and TCR V $35.1,5.2$ analysis. (B) Splenocytes from day 8 PV-infected LCMV-immune mice (LCMV+PV) stained with CD8 and tetramers. Numbers in upper-right corners represent the percentage of gated CD8 T cells binding LCMV NP ${ }_{205}$ and $\mathrm{PV} \mathrm{NP}_{205}$ tetramers; representative of 3 experiments. (C) Histograms of V $35.1,5.2-$ positive cells after gating on CD8 and double tetramer-positive cells. (D and E) Private specificity of $\mathrm{NP}_{205}$ response. Splenocytes from individual LCMVimmune donor mice were transferred into 3 congenic recipients. (D) Numbers in upper-right corners represent the percentage of gated CD8 T cells binding both tetramers 8 days after PV infection. (E) TCR V $\beta$ repertoire of LCMV NP 205 -specific CD8 T cells in LCMV-immune mice before and 8 days after PV infection. Percentage of $\mathrm{V} \beta$ usage was calculated after gating on the IFN- $\gamma$-positive CD8 T cell population. The percentage for other V $\beta$ was calculated by subtracting the sum of the indicated $V \beta$ families from $100 \%$. Numbers in lower bar graphs represent mean percent of $V \beta 5.1,5.2$ usage $(A$ versus B: $P<0.005$; A versus C: $P<0.0002$; $B$ versus $C$ : $P<0.03$ ). In $\mathbf{B}$ and $\mathbf{C}$, letters $A-J$ represent individual mice. In $\mathbf{D}$, letters A-C represent donor mice, and numbers $1-3$ represent recipient mice receiving cells from the specified donor. 

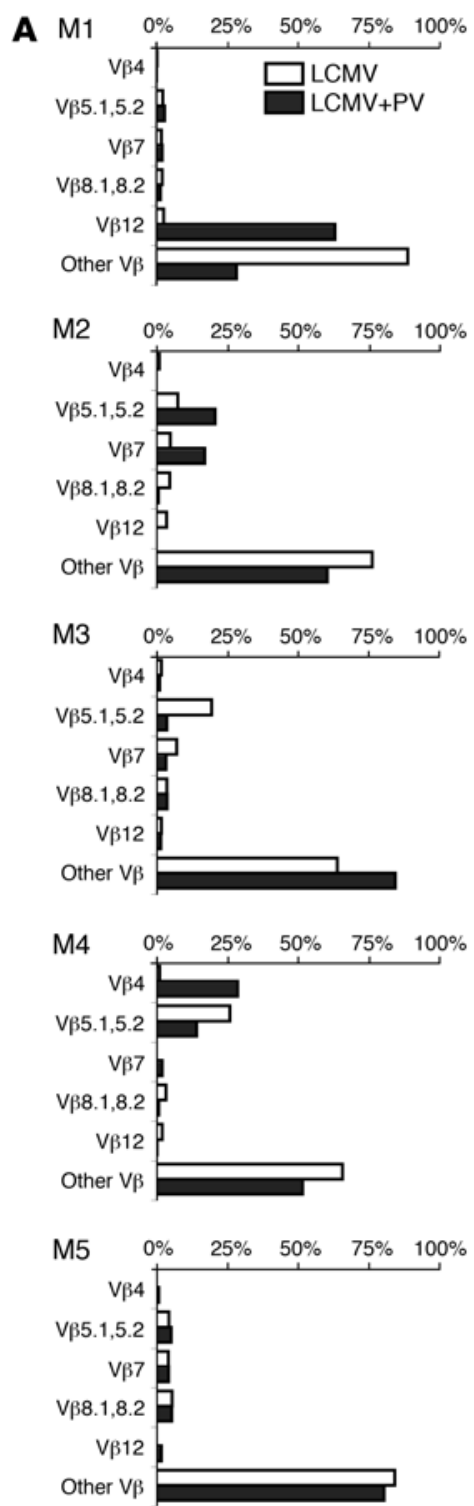
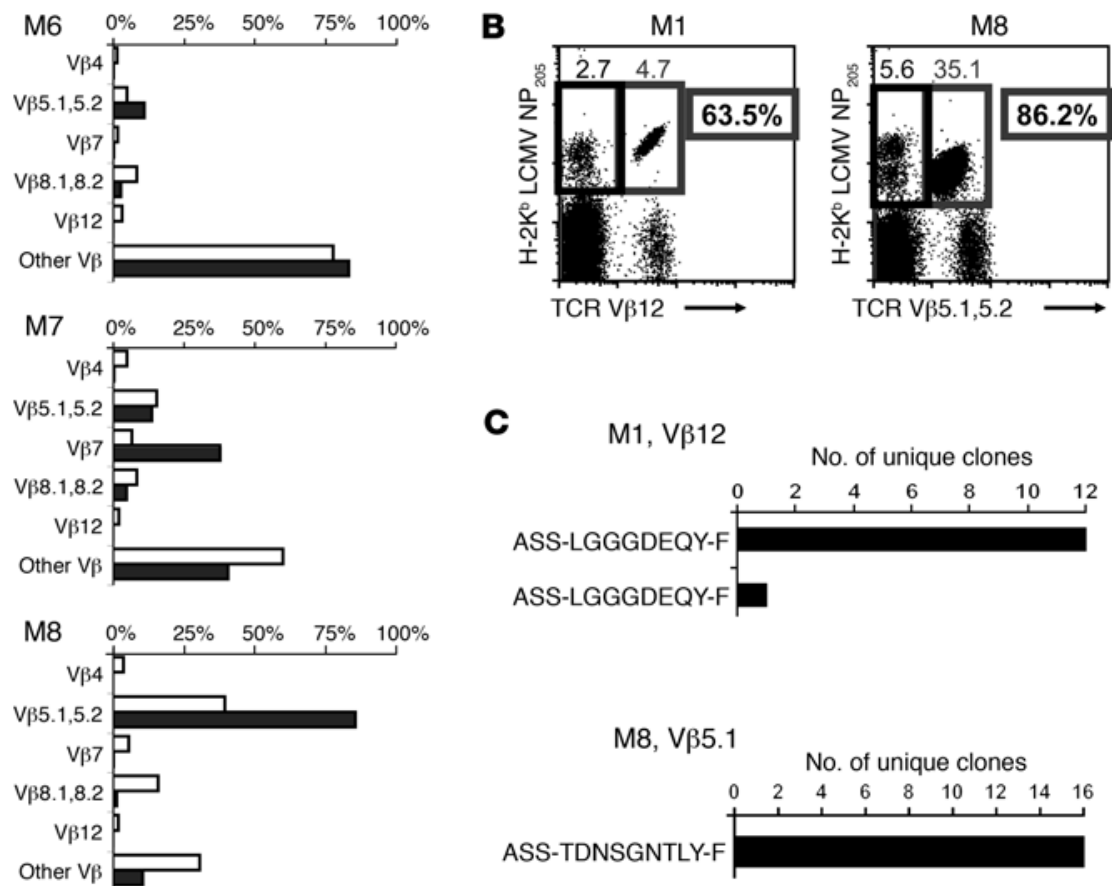

M8, V $\beta 5.1$

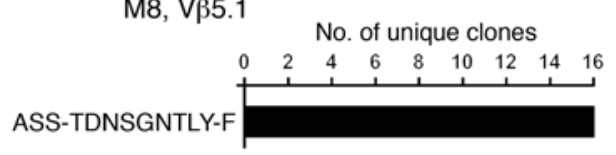

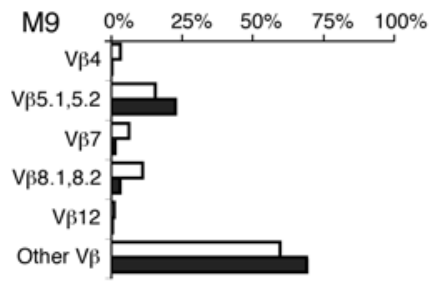

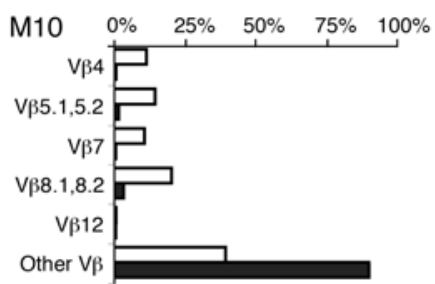

Figure 4

Narrowing of the private LCMV NP 205 -specific CD8 T cell V $\beta$ repertoire after heterologous PV infection. (A) PBMCs were isolated from 10 LCMV-immune mice (white bars), and the LCMV NP ${ }_{205}$-specific CD8 T cells were analyzed either by ICS or by tetramer staining and costaining with $\mathrm{V} \beta$-specific antibodies. The same mice were infected with PV (black bars), and 8 days after infection PBMCs were isolated, and the TCR $\mathrm{V} \beta$ repertoire of $L C M V N P_{205}$ tetramer-positive cells was analyzed. (B and $\mathbf{C}$ ) The cross-reactive $\mathrm{NP}_{205}$-specific TCR repertoire is oligoclonal after heterologous virus infection. (B) The FACS dot plots show the dominant $\mathrm{V} \beta$ usage of the $\mathrm{NP}_{205}$-specific CD8 T cells from 2 representative LCMV+PV-infected mice (mouse 1 [M1] and M8). (C) The LCMV NP $205-$ specific CD8 T cells from M1 and M8 were sorted, and the dominant $\mathrm{V} \beta$ family was subcloned and sequenced.

not shown). These results suggest that $\mathrm{V} \beta 16$ is a major part of the $\mathrm{V} \beta$ repertoire used by $\mathrm{NP}_{205}$-specific CD8 $\mathrm{T}$ cells in both infections (Figure 2A). However, in all mice infected with PV, V $\beta 5.1,5.2$ was a codominant part of the $\mathrm{PV} \mathrm{NP}_{205}$-specific $\mathrm{T}$ cell repertoire. This was significantly different from LCMV infection, where V $\beta 5.1,5.2$ was less dominant and even absent in some mice (PV: $38 \% \pm 18 \%$ versus LCMV: $12 \% \pm 8.0 \% ; n=13$ per group; $P=0.0001)$. Sequence analyses of the V $\beta 16$ (Figure 2E and Supplemental Tables 2 and 3) or V $\beta 5.1$ (Figure 2F and Supplemental Tables 16 and 17) PV NP 205 -specific repertoires of PV-infected mice showed high diversity of TCR usage, similar to the diversity seen with LCMV.
Altered clonal dominance and repertoire narrowing after heterologous virus infection. We questioned how the TCR repertoire evolves under conditions of heterologous virus infection. PV-immune mice infected with LCMV (PV+LCMV mice) had a dominant $\mathrm{NP}_{205}$ response $(13 \% \pm 7.7 \%$ of $\mathrm{CD} 8 ; n=12)$ due to a proliferation of cross-reactive PV NP 205 -specific memory CD8 T cells, as reported previously (21), and the magnitude of the response varied greatly between individual mice $(4.0-30 \% ; n=12)$. Analysis of the PV $\mathrm{NP}_{205}$-specific TCR $\mathrm{V} \beta$ repertoire in $\mathrm{PV}+\mathrm{LCMV}$ mice by $\mathrm{mAb}$ staining revealed variations in patterns of $V \beta$ usage between mice. Only 2 of 12 mice had a V $\beta$ repertoire like that seen in PV-infected mice, 
A
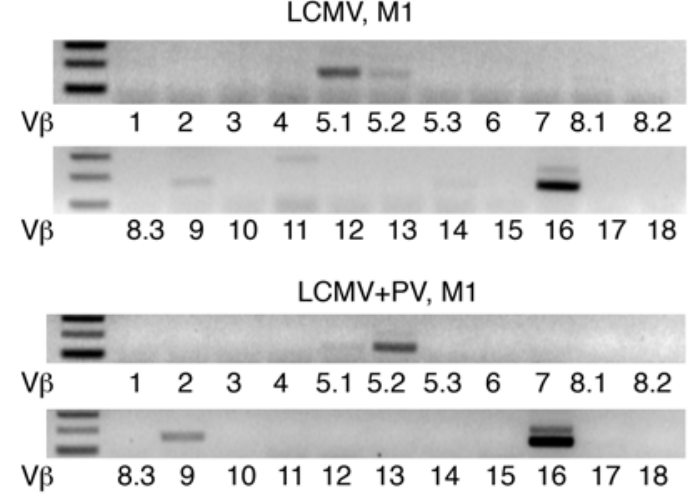

B $\quad \mathrm{V} \beta 16$

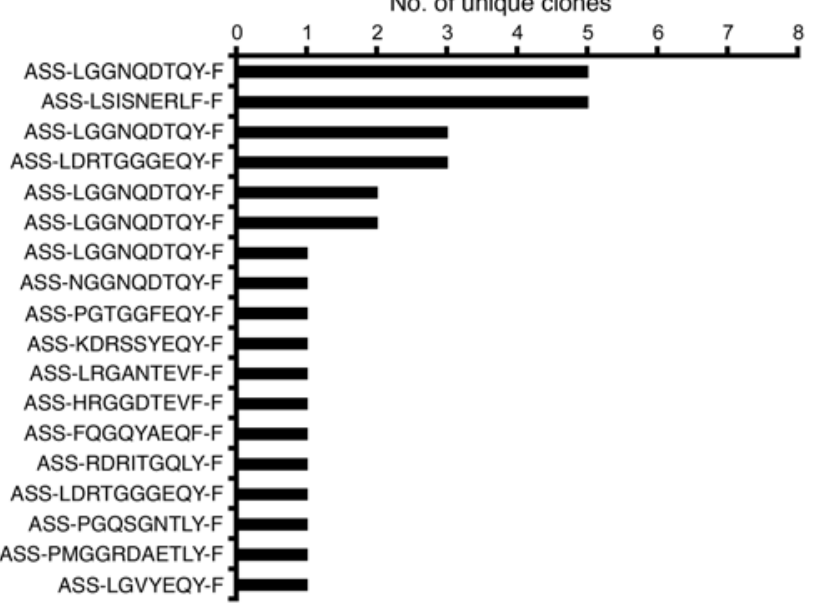

V $\beta 16$

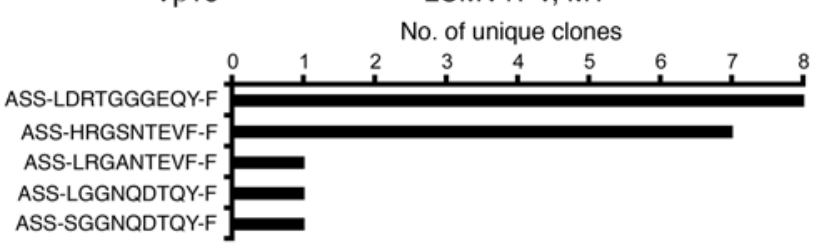

c

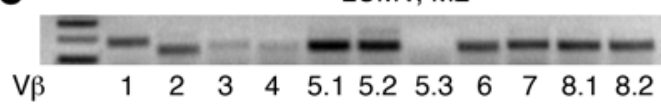

$\mathrm{v} \beta$
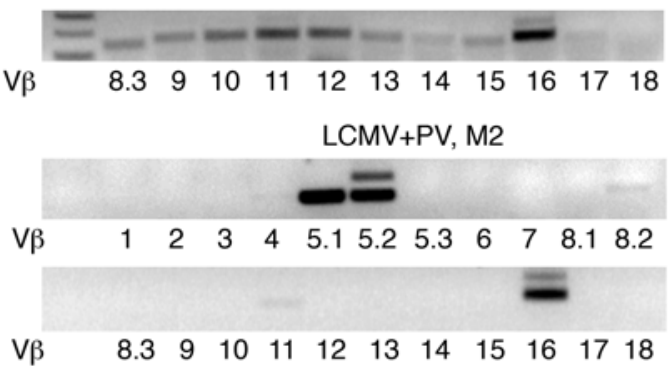

D

V 816

LCMV-immune, M2

No. of unique clones

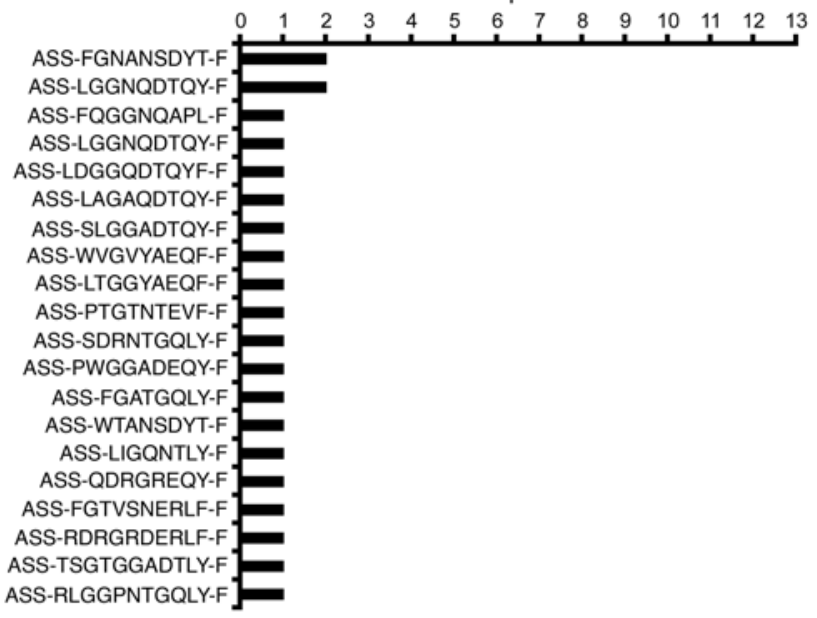

V $\beta 16$

LCMV+PV, M2

No. of unique clones

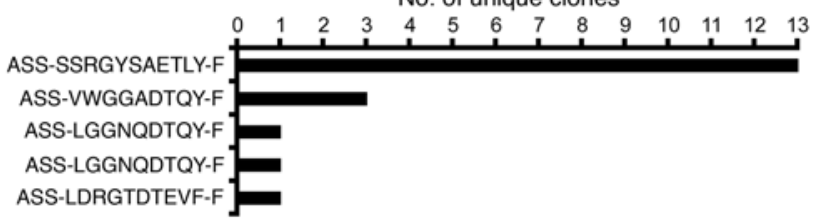

V 35.1

$\mathrm{LCMV}+\mathrm{PV}, \mathrm{M} 2$

LCMV-limmune, M2

No. of unique clones

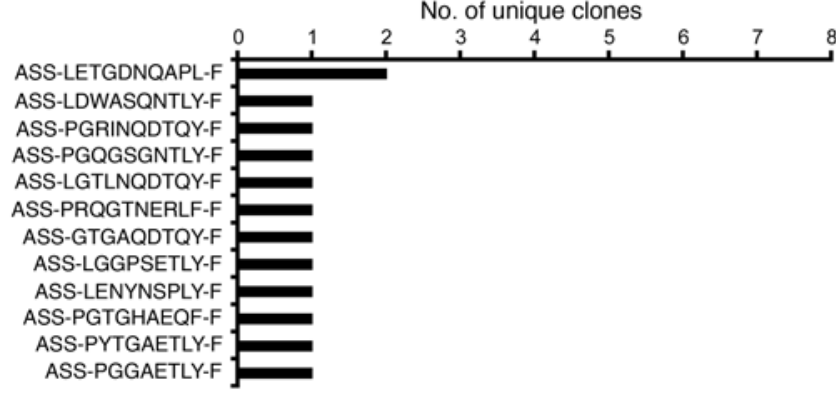

No. of unique clones

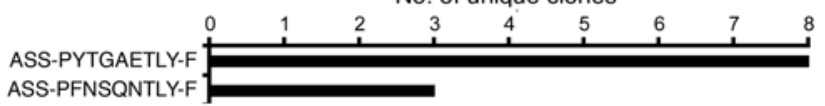

Figure 5

A subset of LCMV NP 205 -specific clones is expanded after heterologous PV infection. PBMCs were from 2 LCMV-immune mice before and 8 days after PV infection. (A and $\mathbf{C}$ ) LCMV NP 205 tetramer-positive CD8 T cells were sorted, and V $\beta$ mRNA expression was analyzed by RT-PCR with specific primers for V $\beta 1-18$. (B, D, and E) The PCR products from dominant V $\beta 16(M 1, \mathbf{A}$ and $\mathbf{B}$; M2, D) and from V $\beta 5.1$ (M2, D) were subcloned, and 13-32 clones were sequenced per group. 
A
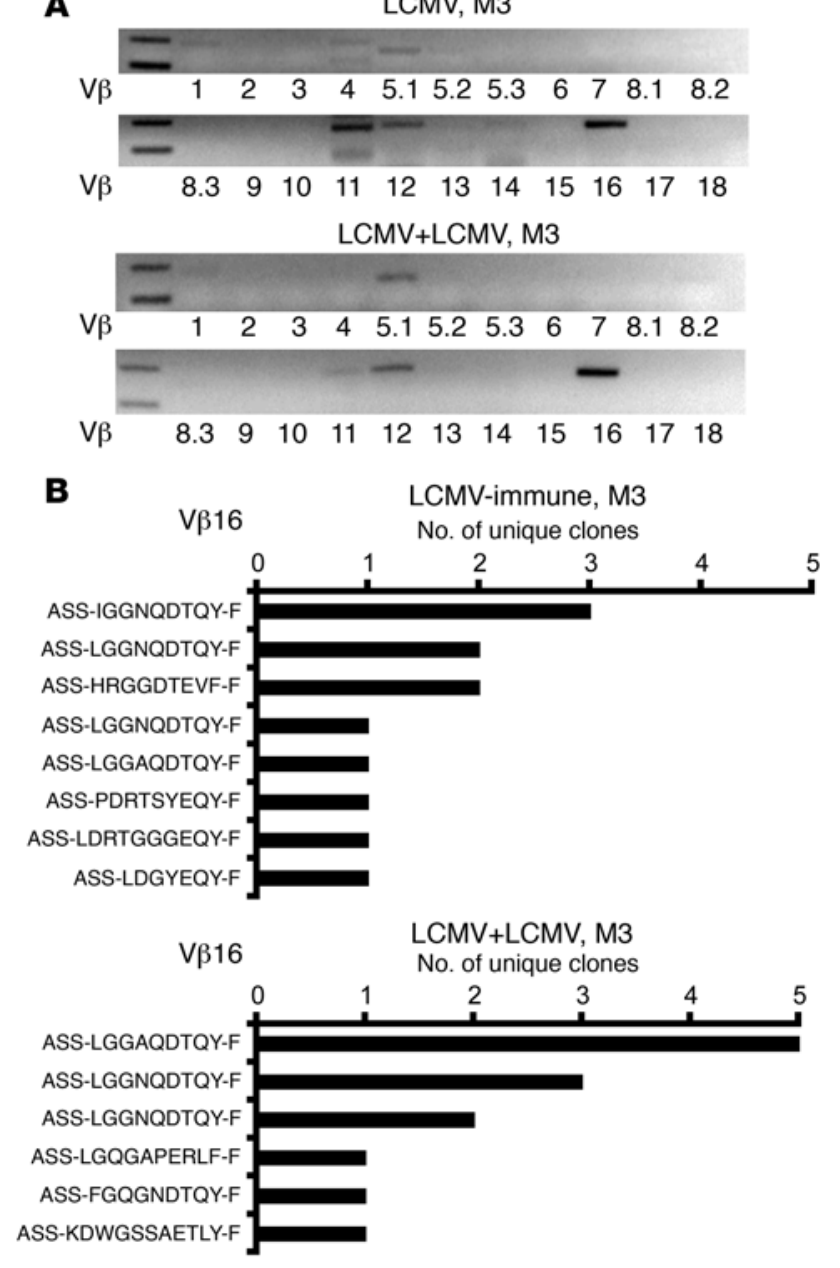

with a $30-40 \%$ V $\beta 5.1,5.2$ usage (Figure $3 \mathrm{~A}$, mouse 1 ). Two other mice had a very high and skewed $V \beta 5.1,5.2$ frequency of $\mathrm{NP}_{205}$-specific CD8 T cells $(56 \%, 61 \%)$. Six of the 12 mice had no dominant $(<25 \%) \mathrm{V} \beta$ usage for the tested mAbs, suggesting a dominant $V \beta 16$ response. Importantly, the $V \beta 5.1,5.2$ frequency was below $5 \%$ in these mice, even though the V $\beta 5$ response is typically higher during the primary PV infection (Figure $2 \mathrm{~A})$. Two other mice demonstrated a dominant and highly skewed response to $V \beta 7$ (89\%; data not shown) and to V $\beta 12$ (69\%; Figure $3 A$, mouse 2$)$. V $\beta 7$ and V $\beta 12$ were never observed as dominant $\mathrm{V} \beta$ families used by $\mathrm{PV} \mathrm{NP}_{205^{-}}$ specific $\mathrm{T}$ cells during the primary $\mathrm{PV}$ infection. Overall, these results suggest that only a subset of the cross-reactive $\mathrm{PV} \mathrm{NP_{205 }}$ CD8 T cells proliferated after heterologous LCMV infection.

In LCMV-immune mice infected with PV (LCMV+PV mice), we similarly observed an enhanced cross-reactive $\mathrm{NP}_{205}$-specific response with high variability in magnitude $(3.0-42 \%$, mean: $15 \% \pm 8.7 \% ; n=24$ ) and in TCR V $\beta$ repertoires (Figure 3, B and C). The $\mathrm{NP}_{205}$ double-tetramer staining of $\mathrm{T}$ cells isolated from individual $\mathrm{LCMV}+\mathrm{PV}$ mice revealed different binding patterns (Figure $3 \mathrm{~B}$ ), and these $\mathrm{LCMV}+\mathrm{PV}$ mice were variable in their TCR $\mathrm{V} \beta$ repertoires (Figure 3C). In 2 of 9 mice (Figure 3C, mice B and E), $75 \%$ of the $\mathrm{LCMV}+\mathrm{PV} \mathrm{NP}{ }_{205}$ response used the V $\beta 5.1,5.2$ family, consistent with only a subset of the $\mathrm{NP}_{205}$-specific CD8 T cells preferentially expanding on PV infection, as V $\beta 5.1,5.2$ was usually only a minor part of the LCMV infection in a naive host (Figure 2A).

\section{Figure 6}

The LCMV NP 205 -specific CD8 T cell repertoire is not skewed after homologous LCMV virus infection. PBMCs were from LCMV-immune mice before and 8 days after LCMV infection. (A) LCMV NP 205 tetramer-positive CD8 T cells were sorted, and V $\beta$ mRNA expression was analyzed by RT-PCR with specific primers for V $\beta 1-18$. (B) The PCR product from dominant $\mathrm{V} \beta 16$ was subcloned, and $12-13$ clones were sequenced per group. This is a representative experiment of 2 .

Private specificities influence patterns of clonal dominance. Adoptive transfer experiments showed that individual variations in the magnitude of the $\mathrm{NP}_{205}$ response and its TCR repertoire were not random events, as memory cells transferred from an LCMV-immune donor mouse into 3 different hosts generated similar double-tetramer staining patterns (Figure 3D) and similar $V \beta$ repertoires (Figure $3 \mathrm{E}$ ) upon PV infection. All recipients from individual donors had statistically similar $V \beta$ repertoires, but recipients from different donors had statistically different $V \beta$ repertoires. The $V \beta 5.1,5.2$ usage in the recipient mice did not correlate with the frequency of LCMV $\mathrm{NP}_{205}$-specific V $\beta 5.1,5.2$ usage of the LCMV-immune donor mouse (Figure 3, D and E), again indicating that only a subpopulation of $\mathrm{T}$ cells proliferated.

Infection with a heterologous virus does not affect the TCR V $\beta$ repertoire of non-cross-reactive epitopes. We asked whether immunity to PV would influence the $V \beta$ usage of the non-cross-reactive $\mathrm{H}-2 \mathrm{D}^{\mathrm{b}}$-restricted epitope LCMV NP 396 . The $\mathrm{NP}_{396}$ response during acute LCMV infection had a dominant V $\beta 8.1,8.2$ TCR repertoire $(n=2: 35 \%, 33 \%)$, consistent with published studies $(14,24)$. This dominance of V $\beta 8.1,8.2$ was not altered in PV-immune mice challenged with LCMV. All mice tested had a dominant (32-52\%, mean: $40 \% \pm 8.0 \%$; $n=7) \mathrm{V} \beta 8.1,8.2$ TCR V $\beta$ repertoire specific to LCMV NP 396 . These data indicate that immunity to another virus does not influence the quality of the response to non-cross-reactive CD8 $\mathrm{T}$ cell epitopes. We made similar observations for the $\mathrm{V} \beta 11$-enriched $\mathrm{H}-2 \mathrm{~K}^{\mathrm{b}}$-restricted epitope LCMV GP ${ }_{34}$ in longitudinal studies of LCMV-immune mice before and after challenge with PV (data not shown).

Longitudinal analysis of T cell repertoire evolution. PBMCs of LCMVimmune mice before and after PV infection were costained with LCMV NP 205 tetramers and V $\beta$-specific mAbs. Most mice used the undetected $V \beta$ family, most likely $V \beta 16$, while some mice had a codominant $\mathrm{V} \beta 5$ response (Figure $4 \mathrm{~A}$ ). The $\mathrm{V} \beta 5$ frequencies in LCMV NP $\mathrm{N}_{205}$-specific $\mathrm{T}$ cells of all tested mice acutely infected with LCMV and LCMV-immune mice were not statistically different $(12 \% \pm 8.0 \%$ versus $13 \% \pm 11 \% ; n=13$ and $n=36$, respectively; $P=0.6$ ), supporting the concept that the T cell repertoire does not differ substantially between the acute response and the memory phase $(14,16,17,25,26)$. However, after heterologous PV infec-

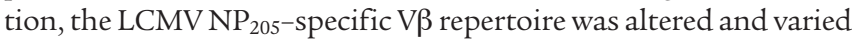
between different mice (Figure 4A).

Mouse 1 had a dominant $64 \%$ V $\beta 12$ usage, and mouse 8 had an $86 \%$ V $\beta 5$ usage of the LCMV NP 205 -specific response (Figure 4B). Subcloning and sequencing of the TCR- $\beta$ third complementarity-determining region (CDR3) of tetramer-sorted cells showed that the LCMV NP 205 response was highly restricted in these mice. Mouse 1 used only $2 \mathrm{~V} \beta$ clones of 13 sequenced for the LCMV $\mathrm{NP}_{205}$-specific V $\beta 12$ response (Figure 4, B and C, and Supplemental Table 21), accounting for two-thirds of the response, and mouse 8 used only a single clone of 16 sequenced (Figure 4, B and C, and Supplemental Table 20), which suggests that more than 
A
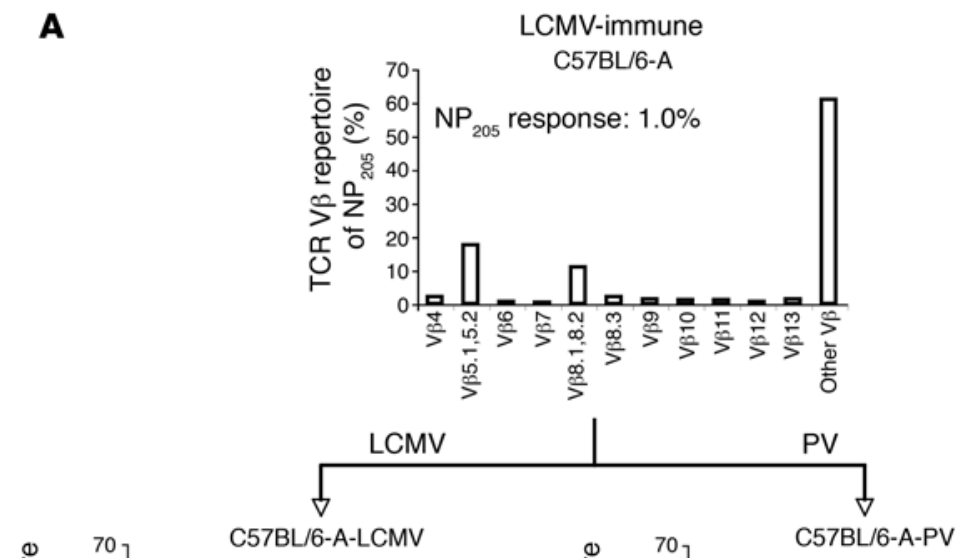

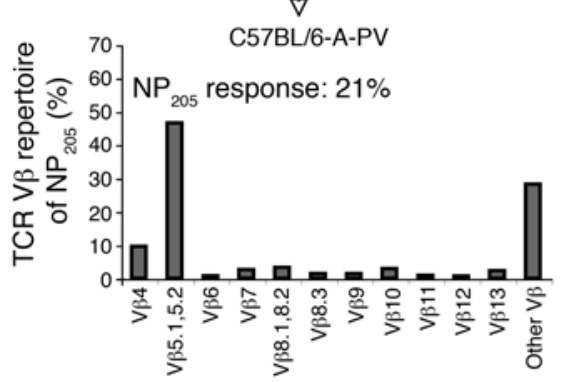

B

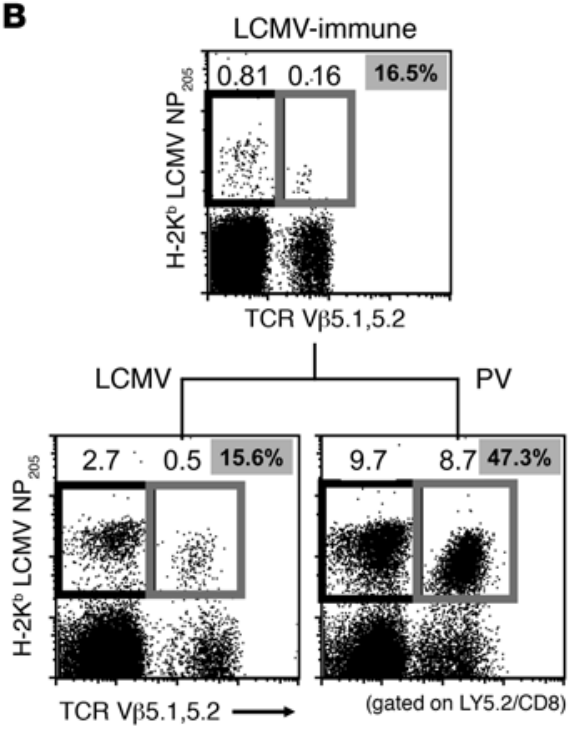

C

$\mathrm{V} \beta 16$

LCMV-immune

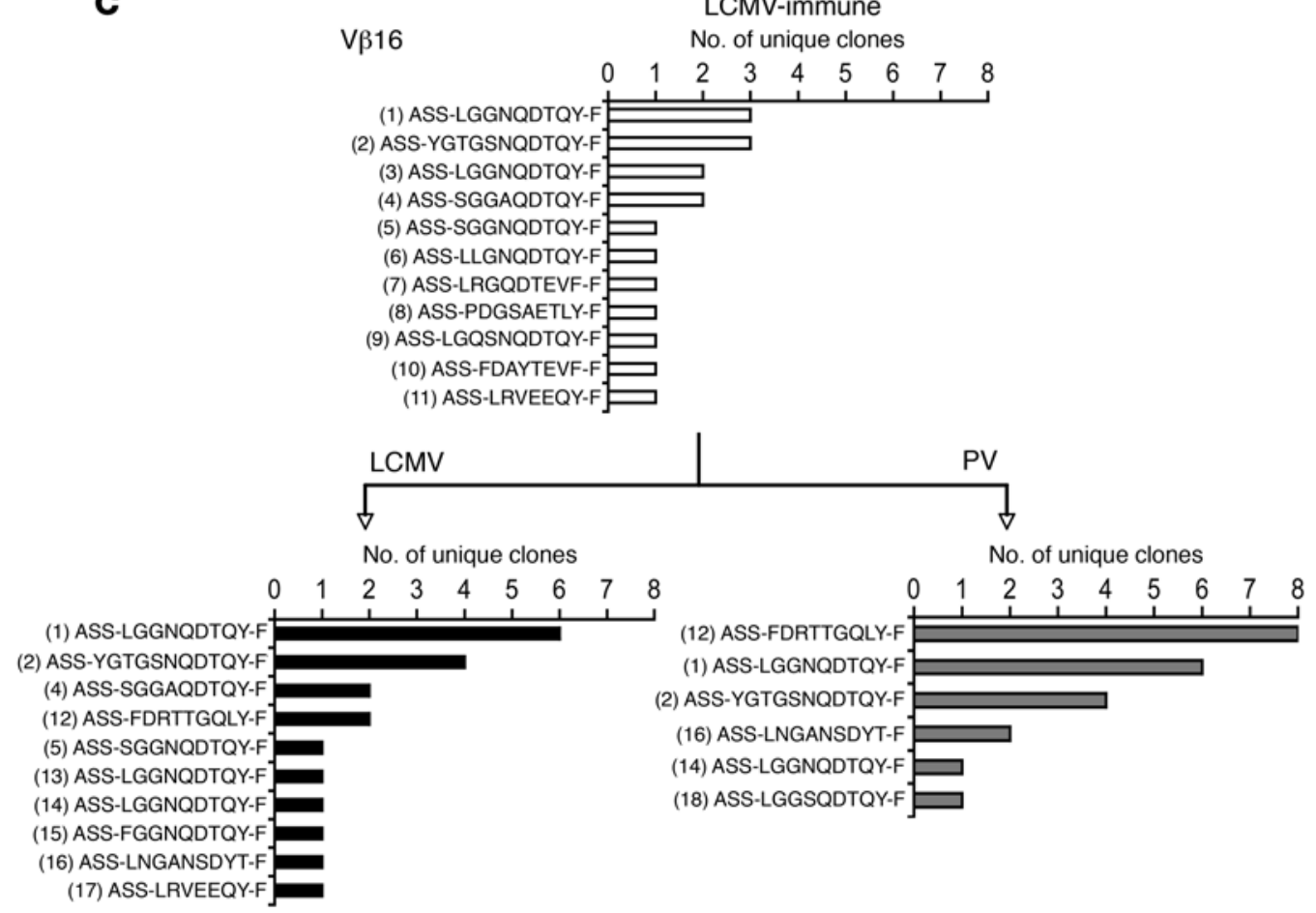

Figure 7

Homologous versus heterologous infection of a private TCR repertoire. Splenocytes from an LCMV-immune donor mouse were transferred into 2 congenic recipients. One recipient was infected with LCMV and the other was infected with PV. (A) TCR V $\beta$ repertoire of NP 205 -specific CD8 T cells. Splenocytes from the LCMV-immune donor and recipient mice 8 days after LCMV or PV infection were stimulated with LCMV NP 205 peptide. The percentage of $\mathrm{V} \beta$ usage was calculated on the gated IFN- $\gamma$-positive CD8 T cell population. $V \beta 17$ served as a negative control. The percentage for other $\mathrm{V} \beta$ was calculated by subtracting the sum of the indicated $\mathrm{V} \beta$ families from 100\%. C57BL/6-A, C57BL/6 donor A. (B) TCR V $\beta$ 5.1,5.2 of LCMV $\mathrm{NP}_{205}$ tetramer-positive CD8 T cells before and after infection. Staining with LCMV NP 205 tetramer and V $\beta 5.1,5.2$ antibody was performed on the CD8 LCMV-immune or congenic donor CD8 T cells (LCMV+LCMV, LCMV+PV). The numbers shown above the gates represent the percentage of cells in the gate. The number in the upper-right quadrant (gray box) represents the percentage of LCMV NP 205 -specific CD8 T cells positive for V $\beta 5.1,5.2$. This $\mathrm{NP}_{205}$-specific $\mathrm{V} \beta 5.1,5.2$ frequency was similar in the ICS assay. Data are from 1 of 5 experiments, where V $\beta 5.1,5.2$ proliferated after PV infection. (C) $\mathrm{V} \beta$ clonotypes after PV or LCMV infection of mice harboring the same memory pool. Splenocytes from LCMV-immune mice were adoptively transferred into 2 recipient mice, which were then infected with LCMV or PV. This experiment used mice different from those represented in $\mathbf{A}$ and $\mathbf{B}$. 


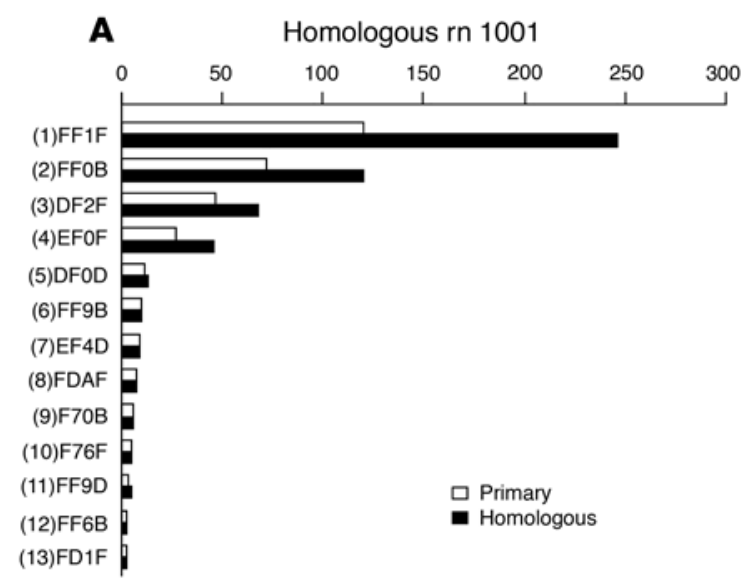

B

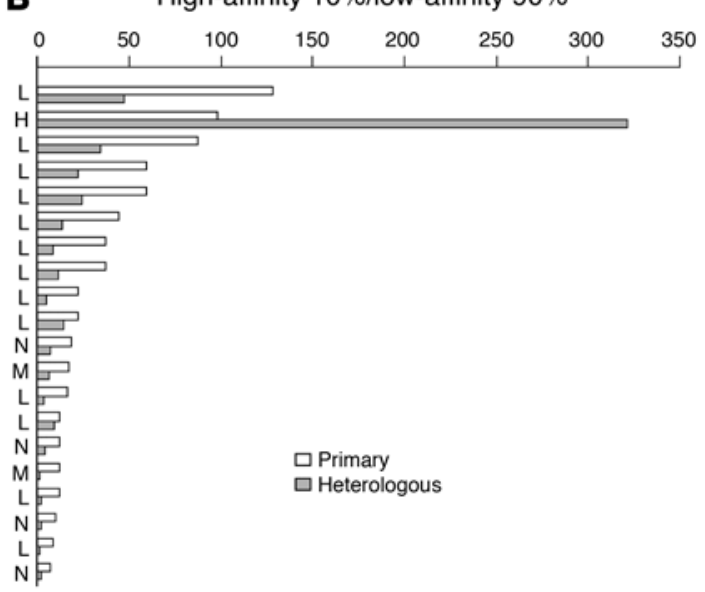

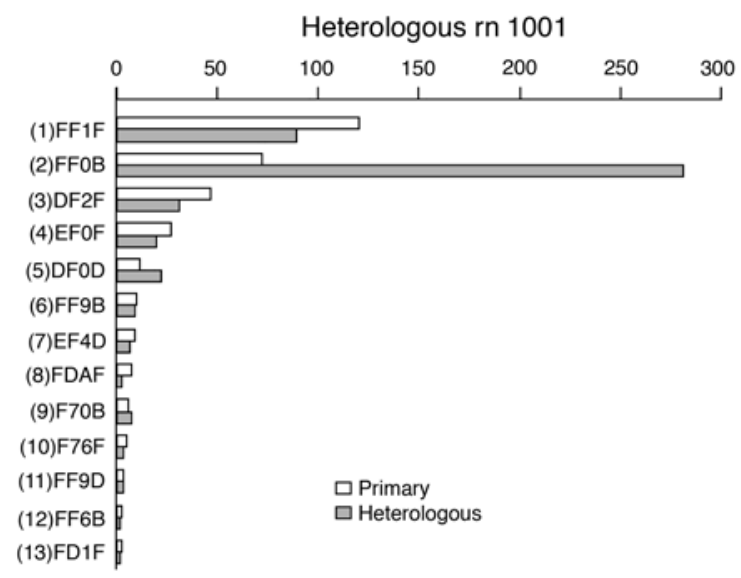

High-affinity $50 \% / l o w-a f f i n i t y ~ 50 \%$

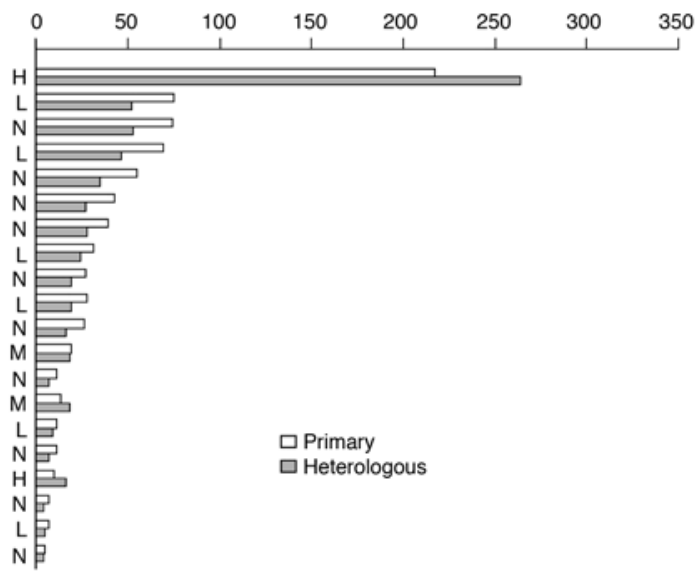

Figure 8

Computer simulation of homologous versus heterologous virus challenge. (A) Left: Clonal distribution of the memory population before (white bars) and after (black bars) a homologous challenge. Right: The same population before (white bars) and after (gray bars) a heterologous challenge. The $x$ axis shows the absolute number of CD8 T cells, while $y$ axis labels indicate hexadecimal representation of each clone involved in the response. Number in brackets on the left of the clone labels represents the place occupied by a particular clone in the immune hierarchy at the end of the primary response. Clones generated after the primary response are ordered according to decreasing cell number. This order is upset when there is a change of hierarchy occurring after the second challenge. rn, random number. (B) Computer simulation of effects of affinity on heterologous virus challenge-induced skewing of the T cell repertoire. Graphs show 2 examples of heterologous challenge in mice selected to possess a memory population with a defined percentage of high/low-affinity cells specific for the challenging virus (10\% high/90\% low in the left graph, $50 \%$ high $/ 50 \%$ low in the right graph). White bars represent the memory population after the primary response and before the challenge, while gray bars represent the same population after the heterologous challenge. The $x$ axis shows the size of each clone in terms of absolute cell number. On the $y$ axis labels indicate the affinity of each clone for the heterologous virus: $\mathrm{H}$, high affinity; M, medium affinity; $\mathrm{L}$, low affinity; $\mathrm{N}$, non-cross-reacting (see Methods).

one-third of that mouse's CD8 T cells (41\% LCMV NP 205 and $86 \%$ $\mathrm{V} \beta 5$ ) was composed of only $1 \mathrm{~V} \beta$ clone.

The evolution of the clonal composition of the LCMVimmune $\mathrm{NP}_{205}$-specific TCR repertoire was examined before (Supplemental Tables 4 and 5) and after (Supplemental Tables 10 and 11) PV infection of the same LCMV-immune mouse. Mouse 1 had a strong $\mathrm{V} \beta 16$ usage of the $\mathrm{NP}_{205}$-sorted cells, both before and after the PV challenge (Figure 5A). Figure 5B shows $\mathrm{NP}_{205}$-specific $\mathrm{V} \beta 16$ clonotypes used by mouse 1 before and after PV infection. The V $\beta 16$ repertoire of this LCMV-immune mouse and several others (Supplemental Tables 4-9) revealed a diverse TCR repertoire, with some clones more frequent than others. However, after PV infection, only 2 clonotypes accounted for more than $80 \%$ of the TCRs, suggesting a more skewed and focused repertoire. This contrasted greatly with the high diversity of clonotypes in naive mice acutely infected with PV (Figure 2, E and F, and Supplemental Tables 2 and 3). Formerly lower-frequency or undetected clones in the resting memory repertoire dominated the response after PV infection. Similar observations were made with a second mouse (Figure 5, C-E). LCMV-immune mouse 2 had a poly-V $\beta$ repertoire with a strong $V \beta 5.1,5.2$ signal. After $P V$ infection, the $V \beta$ repertoire became more focused (Supplemental Table 19), as V $\beta 5.1,5.2$ and V $\beta 16$ became dominant (Figure 5C). Subcloning and sequencing the PCR products of V $\beta 5.1$ (Supplemental Tables 18 and 19) and V $\beta 16$ (Supplemental Tables 4, 5, 10, and 11) demonstrated a diverse TCR repertoire before PV infection but a highly skewed repertoire after PV infection (Figure 5, D and E). 


\begin{tabular}{|c|c|c|c|c|}
\hline Infection sequence & $\begin{array}{l}\text { No. of mice } \\
\text { analyzed }\end{array}$ & $\begin{array}{l}\text { No. of } \\
\text { clones }\end{array}$ & $\begin{array}{c}\text { No. of } \\
\text { clonotypes }\end{array}$ & Ratio \\
\hline LCMV & 8 & 144 & 101 & 1.43 \\
\hline PV & 4 & 63 & 44 & 1.43 \\
\hline LCMV+LCMVA & 3 & 58 & 25 & 2.32 \\
\hline LCMV+PV & 6 & 99 & 21 & 4.71 \\
\hline$P V+L C M V A$ & 2 & 41 & 8 & 5.13 \\
\hline
\end{tabular}

ALCMV clone 13

Constancy in the LCMV NP 205 -specific TCR repertoire after homologous virus infection. Two LCMV-immune mice analyzed before (Supplemental Tables 6 and 7) and after (Supplemental Tables 13 and 14) homologous LCMV (LCMV variant clone 13) challenge had diverse $V \beta 16$ repertoires in each case (Figure 6). Clone 13 was used because it grows better in vivo, but its T cell epitopes are identical to those of the Armstrong strain and, where tested, induce similar TCR repertoires. In several LCMV-immune mice tested, the $\mathrm{V} \beta 16$ repertoire included many clones of low frequency, but many used the joining region of TCR $\beta$-chain 2.5 (J $\beta 2.5$ ), depicted by the sequence X-QDTQY-F, and expressed a CDR3 region amino acid motif of XGGNorA (Supplemental Tables 1 and 4-9). These sequences (LGGAQDTQY, LGGNQDTQY) still dominated after homologous virus infection, suggesting a selection for this CDR3 motif (Figure 6, A and B). Together with data from other studies $(14,16,17,26)$, our data indicate that the level of TCR diversity is not dramatically different between primary and homologous secondary immune responses for nonpersistent pathogens, though there was a slight skewing toward the dominant motif. This contrasts with the marked skewing seen after heterologous virus challenge (Figure 2D, Figure 5, B and E, and Figure 6B), which may completely alter and distort the TCR repertoire against cross-reactive epitopes.

Homologous versus heterologous challenge of the same T cell repertoire. To show that the same private TCR repertoire of the $\mathrm{NP}_{205}$ population of an LCMV-immune mouse would skew differently, depending on a homologous versus heterologous challenge, splenocytes from $1 \mathrm{LCMV}$-immune donor mouse were transferred into 2 congenic recipient mice, which were then infected with LCMV or PV. The $\mathrm{NP}_{205}$-specific $\mathrm{V} \beta$ repertoire of this LCMV-immune mouse was similar before and after secondary LCMV infection but was very different after PV infection, with a dramatic increase in $\mathrm{NP}_{205}$ frequency and skewing of the repertoire to $\mathrm{V} \beta 5.1,5.2$ (48\%) (Figure 7, A and B). In a similar experiment, we compared the clonal composition of the LCMV NP $\mathrm{N}_{205}$-specific $\mathrm{V} \beta 16$ repertoire before and after challenge with either LCMV or PV (Figure 7C and Supplemental Tables 9, 12, and 15). In this mouse, V $\beta 16$ was the dominant family before and after infection with LCMV or PV. However, after heterologous PV infection, the hierarchy of the clones skewed and shifted, and a clone originally of lower frequency dominated (Figure 5B).

Simulation by computer modeling. The data showing the narrowing of the repertoire on heterologous virus challenge were simulated by computer modeling of the immune system in a "virtual" mouse. The private specificities of mice were reproduced by utilizing a sufficiently large diversity of TCRs, which allowed us to obtain and study the characteristic memory populations and their growth and/or modification in hierarchy after homologous and heterologous challenge. Figure 8A shows a representative experiment comparing homologous versus heterologous challenge out of 30 experiments conducted, where focusing of the clonal distribution was monitored by the skewness index increase. These computer-generated data are remarkably similar to the biologically determined data with LCMV and PV shown in Figure 8A and allow us to state the following: (a) homologous challenge only weakly focuses the repertoire; the skewness of the memory population before (1.71) and after (1.76) the homologous challenge is not statistically different; (b) heterologous challenge results in altered clonal dominance and a significant narrowing of the preexistent memory distribution, and the skewness after challenge (2.29) is significantly higher than before challenge $(1.71 ; P<0.01)$; (c) there is a significant difference in the average skewness of 30 homologous challenges and 30 heterologous challenges of the same memory population, with the index being higher in the heterologous $(P<0.01)$.

To study in silico the effect of clonal affinity for the heterologous peptide on the clonal population changes, we constructed memory $\mathrm{T}$ cell repertoires with a number of same-sized clones (e.g., 30 cells) representing different mixtures of affinity levels. Virtual mice were primed and programmed to show $10 \%$ high $/ 90 \%$ low- or $50 \%$ high $/ 50 \%$ low-affinity cells toward the heterologous peptide (Figure $8 \mathrm{~B}$ ). The conclusions of these experiments are that high affinity clones have the highest probability of reaching a dominant position after challenge, although both the competition and the low initial representation can thwart their chances. It also indicates that more skewing may occur if there is initially a smaller rather than larger proportion of high affinity cross-reactive clones.

Generation of an epitope escape mutant. Some correlations have been made between the presence of oligoclonal TCR repertoires and the generation of $\mathrm{T}$ cell epitope escape variants in human viral systems. To directly test under controlled conditions whether a narrow repertoire might yield an escape variant, mice immune to PV were challenged with the clone 13 strain of LCMV, which causes persistent infections and narrows the $\mathrm{NP}_{205}$ repertoire (Table 1). Eight months after infection, most virus was cleared, but a variant in the $\mathrm{NP}_{205}$ epitope was isolated from the kidney, the only tested organ where virus was found. This had a $\mathrm{V} \rightarrow \mathrm{A}$ change in the third position of the epitope (Figure 9A). Of note is that the wild-type epitope sequence is well conserved, and all of 8 sequenced New World arenaviruses have valine in the third position. However, the Old World arenaviruses Lassa and Mopeia have alanine in the third position, indicating that this can be perpetuated in nature. Both wild-type and mutant peptides stabilized $\mathrm{K}^{\mathrm{b}}$ on the surface of RMA-S cells, which normally do not express surface class I MHC due to a mutation in the class I peptide transporting Tap-1 protein. This indicates that the mutant epitope had the potential to be presented to T cells (Figure 9B). However, the $\mathrm{V} \rightarrow \mathrm{A}$ peptide was less effective than the wild type at stimulating IFN- $\gamma$ from $T$ cells from either LCMV-infected (data not shown) or PV+LCMV-infected mice (Figure 9C). Infection of mice with the mutant $V \rightarrow A$ virus resulted in normal CD8 $\mathrm{T}$ cell responses to the immunodominant epitopes GP33, NP396, and GP276, but a substantially reduced response to either wild-type or mutant $\mathrm{NP}_{205}$ epitopes (Figure 9D). To our knowledge, this is the first reported escape mutant in the LCMV NP 205 epitope. 
A

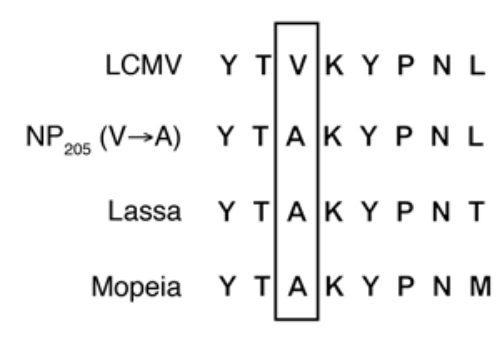

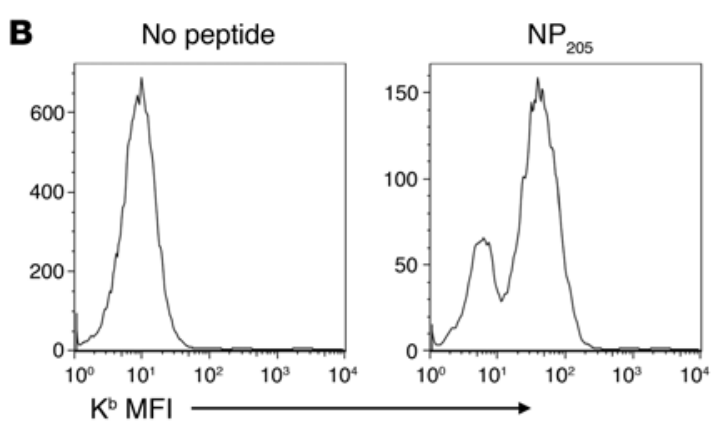

C

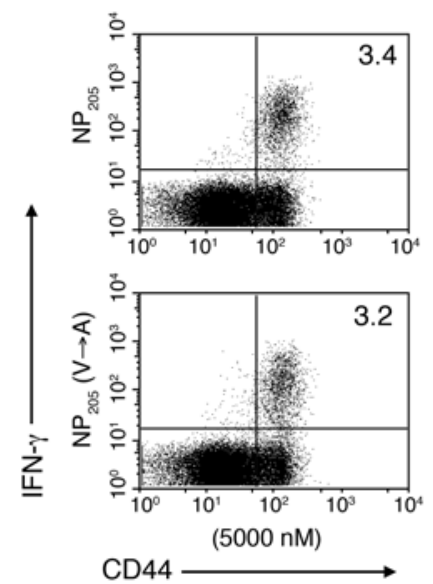

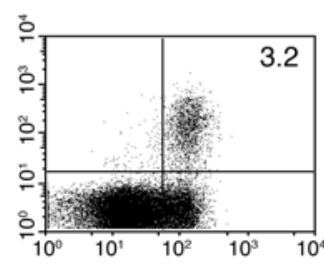

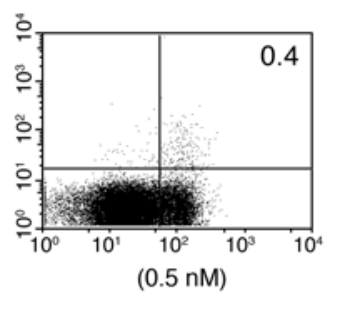

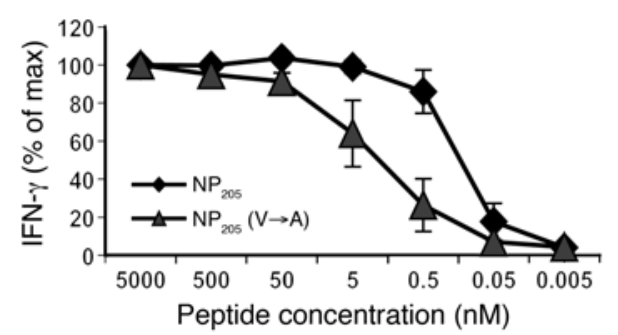

D

Day 8 LCMV effectors

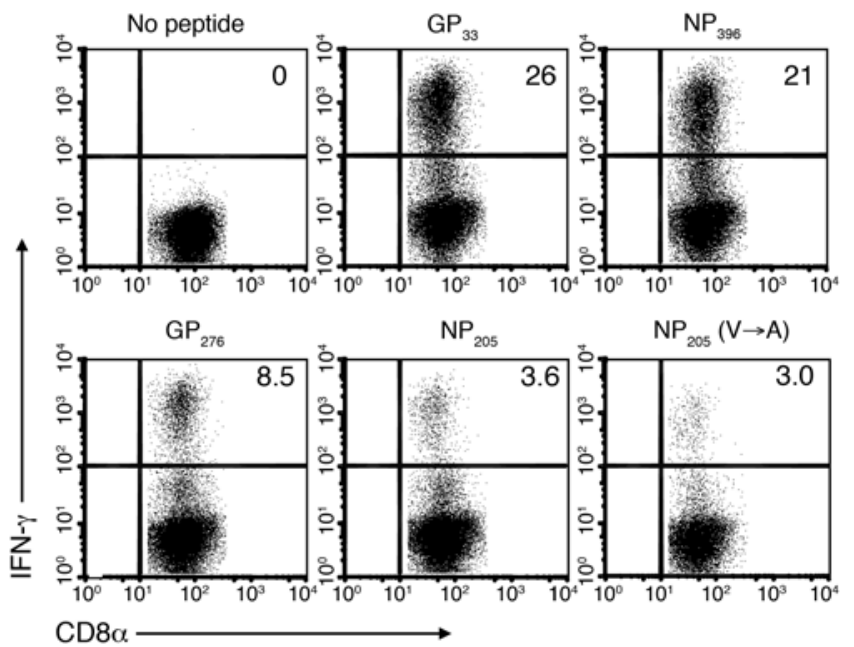

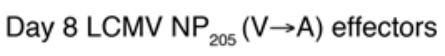
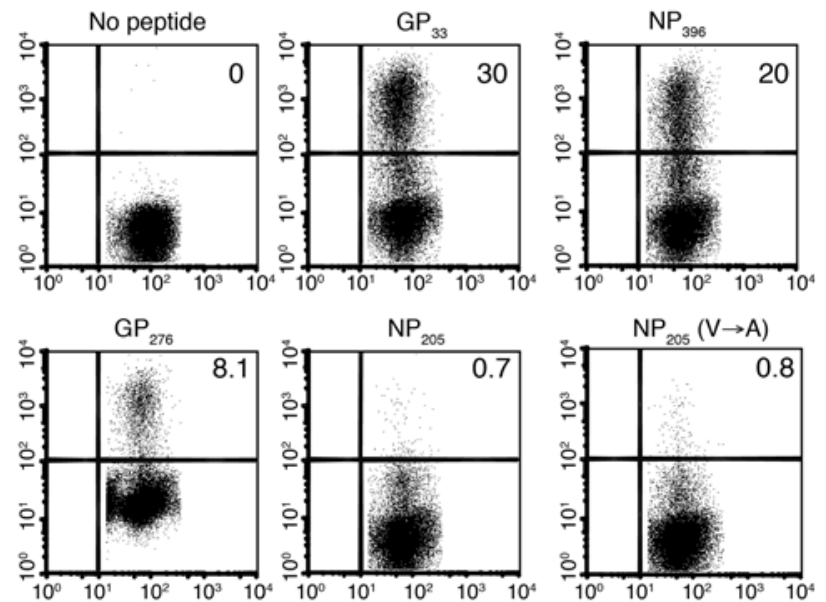

\section{Figure 9}

Characterization of the $\mathrm{NP}_{205}(\mathrm{~V} \rightarrow \mathrm{A})$ variant. $(\mathbf{A}) \mathrm{NP}_{205}$ epitope sequences from Old World arenaviruses. (B) Peptide MHC stabilization assay. RMA-S cells were incubated with $100 \mu \mathrm{M}$ of the indicated peptides overnight. The $y$ axis indicates the mean fluorescence intensity (MFI) of the anti- $\mathrm{K}^{\mathrm{b}} \mathrm{mAb}$ staining. Data are representative of 2 experiments. (C) Peptide titration. IFN- $\gamma$ production of splenocytes from PV-immune + LCMV mice $(n=3)$ in response to serial dilutions of LCMV NP 205 wild-type and LCMV NP $205(\mathrm{~V} \rightarrow \mathrm{A})$ variant peptides. A representative FACS plot with stimulation of $5,000 \mathrm{nM}$ and $0.5 \mathrm{nM}$ of peptides is shown. Numbers in the upper-left corners represent the percentage of CD44hi $T$ cells producing IFN- $\gamma$. IFN- $\gamma$ responses are plotted as percentage of the maximal response to each peptide stimulation. Differences between LCMV NP 205 wild-type and LCMV NP $205(\mathrm{~V} \rightarrow \mathrm{A})$ were statistically significant $(P=0.0045)$ at $0.5 \mathrm{nM}$. (D) Virus-induced CD8 T cells. Splenocytes from mice inoculated with either LCMV $(n=2)$ or LCMV NP $205(\mathrm{~V} \rightarrow \mathrm{A})$ variant virus $(n=3) 8$ days after infection were stimulated with the indicated peptides in an intracellular IFN- $\gamma$ assay. Numbers in the upper-left quadrants represent the percentage of CD8 T cells producing IFN- $\gamma$.

\section{Discussion}

Here we examined the evolution of the TCR repertoires specific to highly cross-reactive epitopes of different viruses. Despite identity in the amino acids emanating from the MHC, these epitopes induced different and diverse TCR repertoires, yet most T cells from each repertoire synthesized IFN- $\gamma$ in response to the other epitope. Striking differences in repertoire development, however, were noted when mice received a homologous versus heterologous 
viral challenge. The results of all of our sequence analyses shown in the text and in the Supplemental Figures are summarized in Table 1, which lists the number of molecular $V \beta$ clones sequenced and the number of different sequences (clonotypes) they represent. Infections with either LCMV or PV alone induced very broad repertoires, with most analyzed clones represented only once and each with a clone/clonotype ratio of 1.43 . Homologous LCMV challenge only slightly narrowed the repertoire (to a ratio of 2.32), and in each case, the repertoire predictably narrowed toward an already dominant V $\beta 16$ J $\beta 2.5$ CDR3 motif of XGGNorA (Figure 6 and Supplemental Tables 13-15). Heterologous challenge in either virus sequence led to profound narrowing of the repertoire (to 5). Under these conditions of heterologous infection, the V $\beta$ usage and CDR3 motifs were highly skewed, unpredictable, and a function of the private specificity of the host's unique $\mathrm{T}$ cell repertoire (Figures 4, 5, and 7C, and Supplemental Tables 10-12 and 19-21). Thus, extremes in clonal dominance and oligoclonality can be a function of heterologous immunity.

Computer studies with immune virtual mice challenged with a cross-reactive epitope mimicked our experimental data showing that $\mathrm{T}$ cell cross-reactivity can modulate clonal dominance and narrowing of the TCR repertoire (Figure 8A). This system was further useful in predicting that repertoire narrowing could be a function of the proportion of high-avidity cross-reactive $\mathrm{T}$ cells (Figure 8B). It is noteworthy that a recent report indicates that high-avidity $\mathrm{T}$ cell populations are selected during persistent human CMV infection (27).

Elements shown to influence epitope-specific immunodominance hierarchies include the efficiency of peptide processing, the affinity of the peptide for the presenting MHC molecule, the overall number of peptide-MHC complexes, the availability of a TCR repertoire able to recognize the peptide-MHC complex, and the phenomenon of immunodomination, where $T$ cells specific for certain immunodominant epitopes suppress responses to other epitopes (28). What causes TCR clonal selection within an epitopespecific response is poorly understood (12), but the remarkable repertoire skewing after a heterologous versus homologous viral challenge is likely a consequence of the subtle avidity differences of different clones of $\mathrm{T}$ cells to the heterologous epitope and the slower clearance of the heterologous viral antigens, which allows for a longer time period to drive selection of the repertoire.

Cross-reactivity may explain why $\mathrm{T}$ cell responses to some epitopes in human viral infections have a narrow oligoclonal TCR repertoire, while others are diverse. A narrowed repertoire with a high-affinity clone in some circumstances may help to control a pathogen early in infection (29), but if the pathogen is not efficiently cleared, the resultant narrowed repertoire might enable mutant viruses to escape the immune system. During acute HIV infection, antigen-specific $T$ cells are associated with the decline of viremia, but narrow CD8 $\mathrm{T}$ cell responses against a single epitope correlate with the generation of HIV T cell escape variants (11). Limited CD8 TCR repertoire diversity has also been correlated with the appearance of $\mathrm{T}$ cell epitope escape variants in chimpanzees with chronic hepatitis $C$ infection (8). T cell epitope escape mutants in the LCMV system have been generated by the unnatural process of cultivating virus-infected cells in vitro in the presence of epitope-specific $\mathrm{T}$ cell clones or by passaging virus in mice harboring transgenic T cells $(30,31)$. Here we were able to isolate an epitope mutant under the far more natural conditions of a heterologous viral challenge and viral persistence (Figure 9A). The mutation was in a nonanchoring position, and, despite the fact that the peptide could be presented by the MHC (Figure 9B), it was not well recognized by $T$ cells (Figure 9, C and D). It is noteworthy that this $\mathrm{V} \rightarrow \mathrm{A}$ mutation made LCMV more like Lassa virus, a related and highly virulent arenavirus of West Africa.

The private specificities of cross-reactive TCR repertoires might explain why some patients have a higher extent of viral escape than others (32). Cross-reactivity between CD8 T cells specific for epitopes of $\mathrm{HCV}\left(\mathrm{NS}_{1073}\right)$ and influenza $\mathrm{A}\left(\mathrm{NA}_{231}\right)$ has been documented (33), and CD8 T cells specific to the cross-reactive epitope $\mathrm{HCV} \mathrm{NS3} 3_{1073}$ are observed during acute $\mathrm{HCV}$ infection (34-36). Notably, HCV NS3 $3_{1073}$ is an epitope for which viral escape mutants have been demonstrated (37), and dominant responses to this cross-reactive epitope unique to the private specificities of an individual have been correlated with rare cases of fulminant acute HCV infection (38). These patients developed a chronic $\mathrm{HCV}$ infection, even though patients with symptomatic $\mathrm{HCV}$ infection are usually more likely to resolve infection due to a strong immune response (39).

These results also have implications for vaccine design, as vaccines inducing the proliferation of cross-reactive memory CD8 $\mathrm{T}$ cells may lead to restricted TCR repertoires that differ between individuals and have different pathogenic outcomes.

\section{Methods}

Mice. C57BL/6 (B6, H-2 ${ }^{\mathrm{b}}$ ) male mice were from the Jackson Laboratory, and B6.SJL-ptprc ${ }^{\text {a }}$ (it.1) congenic male mice were from Taconic. Mice were used at 2-12 months of age and maintained under specific pathogen-free conditions at UMMS. All animal work was reviewed and approved by the UMMS institutional animal use committee.

Virus infection protocol. LCMV (Armstrong strain and variant clone 13) and PV (AN3739 strain) were propagated in BHK21 cells (40). For primary infections, mice were inoculated i.p. with $5 \times 10^{4} \mathrm{PFU}$ LCMV Armstrong or $2 \times 10^{7} \mathrm{PFU}$ PV. Mice were considered immune 6 weeks or later after infection. For homologous challenge experiments, LCMV-immune mice were infected i.p. with $2 \times 10^{6} \mathrm{PFU}$ LCMV variant clone 13 (LCMV+LCMV) to generate a highly disseminating LCMV infection with high virus titers. For heterologous virus challenge, LCMV-immune mice were infected i.p. with $2 \times 10^{7} \mathrm{PFU}$ PV $(\mathrm{LCMV}+\mathrm{PV})$, and PV-immune mice were infected i.p. with $4 \times 10^{5} \mathrm{PFU}$ LCMV Armstrong (PV+LCMV). For isolation of an epitopeescape variant, $\mathrm{PV}$-immune mice were inoculated with $2 \times 10^{5} \mathrm{PFU}$ LCMV clone 13 , and virus was isolated from the kidney 8 months later. To control for culture contaminants, PV stocks were purified through a sucrose gradient and diluted in HBSS (Invitrogen Corp.), and LCMV Armstrong was diluted more than 40-fold in HBSS.

Synthetic peptides. LCMV epitope $\mathrm{NP}_{205-212}, \mathrm{~K}^{\mathrm{b}}$ (YTVKYPNL) (41) and PV epitope $\mathrm{NP}_{205-212}, \mathrm{~K}^{\mathrm{b}}$ (YTVKFPNM) (21) were used in this study. Other LCMV epitopes were $\mathrm{NP}_{396-404}$ (FQPQNGQFI), GP 33-41 $_{\text {(KAVYNFATC), and }}$ $\mathrm{GP}_{276-286}$ (SGVENPGGYCL) (31) and the $\mathrm{NP}_{205-212} \mathrm{~V} \rightarrow$ A mutant YTAKYPNL. Synthetic peptides were from BioSource International or 21st Century Biochemicals and purified to $90 \%$ purity.

Cell surface and tetramer staining by flow cytometry. Single-cell suspensions of splenocytes or blood lymphocytes were stained as described previously (42) using peridinin chlorophyll protein-anti-mouse (PerCP-anti-mouse) CD8- $\alpha$ (clone 53-6.7) and FITC-anti-CD44 (clone IM7) or FITC-labeled $\mathrm{V} \beta$-specific mAbs (V $\beta 2-14, \mathrm{~V} \beta 17^{\mathrm{a}}$; BD Biosciences - Pharmingen kit). $\mathrm{V} \beta 17^{\mathrm{a}}$ served as negative control. For (double-)tetramer staining, cells were incubated first with streptavidin and Fc block to prevent nonspecific binding, washed, and then stained with PE- and/or allophycocyanin-labeled (APC-labeled) tetramers for 60 minutes. After 40 minutes of tetramer 
incubation, surface antibodies were added for 20 minutes, and cells were washed twice with FACS buffer and fixed in Cytofix (BD Biosciences Pharmingen). Samples were analyzed with a BD FACSCalibur flow cytometer and FlowJo software version 6.X (Tree Star Inc.). All surface antibodies were purchased from BD Biosciences - Pharmingen. MHC class I peptide tetramers specific for LCMV NP $205 / \mathrm{K}^{\mathrm{b}}$, LCMV NP ${ }_{396} / \mathrm{D}^{\mathrm{b}}$, LCMV GP $34 / \mathrm{K}^{\mathrm{b}}$, and PV NP $205 / \mathrm{K}^{\mathrm{b}}$ were generated as described previously (43).

ICS. Spleen or blood $\left(10^{6}\right)$ leukocytes were stimulated either with medium or $5 \mu \mathrm{M}$ peptide unless otherwise indicated (42). In experiments analyzing the $\mathrm{NP}_{205}$-specific IFN- $\gamma$ production and TCR V $\beta$ usage, there was downregulation of the TCR, and therefore a higher concentration of the V $\beta$ mAbs was used $\left(2 \mu \mathrm{g}\right.$ per $10^{6}$ cells in $100 \mu \mathrm{l}$ instead of $\left.0.5 \mu \mathrm{g}\right)$. Intracellular cytokine-producing cells were detected with PE- labeled anti-mouse IFN- $\gamma$ or IgG isotype control mAbs (BD Biosciences - Pharmingen).

Adoptive transfer of LCMV-specific T cells into mice. Spleen leukocytes isolated from LCMV-immune B 6 mice were adoptively transferred via the tail vein into LY5. $1^{+}$B 6 congenic mice. One day after transfer, mice were either infected i.p. with $4 \times 10^{5} \mathrm{PFU}$ LCMV Armstrong or $2 \times 10^{7} \mathrm{PFU}$ PV. Donor T cells were analyzed 6 days after infection using PerCP-anti-mouse CD45.2 (LY5.2, clone 104) and PE- or APC-anti-mouse CD8- $\alpha$ (clone 53-6.7).

TCR V $\beta$ analysis of $N P_{205}$-sorted CD8 T cells by RT-PCR and sequencing of the $T C R V \beta$ chain region. For longitudinal experiments, blood (0.3-0.4 ml) was collected from mice, and for adoptive transfer experiments splenocytes were analyzed before and after transfer. $\mathrm{H}-2 \mathrm{~K}^{\mathrm{b}} \mathrm{LCMV} \mathrm{NP} \mathrm{2}_{205}$ tetramer-positive and tetramer-negative CD8 T cells were sorted with a FACSVantage cell sorter (BD). Tetramer-positive cells (5,000-60,000 cells) were collected into medium containing $2 \times 10^{5}$ cos- 7 monkey kidney cells, which were used as carrier cells. RNA was isolated with TRIzol reagent (Invitrogen Corp.) according to the manufacturer's protocol. Total RNA was transcribed into cDNA with Superscript III reverse transcriptase and oligo-d $\mathrm{T}_{12-18}$ primer (Invitrogen Corp.). TCR V $\beta$ analysis was performed either by a standard qualitative PCR or by spectratype analyses (3) with modifications using HotMaster Taq DNA polymerase (Eppendorf) and specific primers for mouse V $\beta 1-18$ (V $\beta 1$ : 5' -CAGACAGCTCCAAGCTACTTTTAC-3', V $\beta 2$ : 5' - ATGAG C CAGGG CAGAACCTTGTAC-3', Vß3: 5' GAAATTCAGTCCTCTGAGGCAGGA-3', Vß 3 : 5' $5^{\prime}$-CTAAAGCCTGATGACTCGGCCACA-3', Vß5.1: 5'-CCTTGGAGCTAGAGGACTCTGCCG-3',

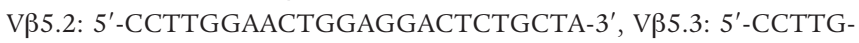
GACCTAGAGGACTTTACTG-3', Vß6: 5'-GCCCAGAAGAACGAGATG-

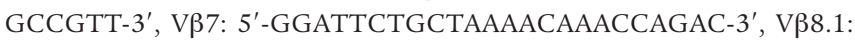
5'-GCTTCCCTTTCTCAGACAGCTGTA-3', Vß8.2: 5' -GCTACCCCCTCTCAGACATCAGTG-3', Vß8.3: 5'-GGCTTCTCCCTCTCAGACATCTT-3',

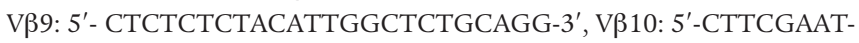
CAAGTCTGTAGAGCCGG-3', Vß11: 5'-TGAAGATCCAGAGCACGCGGCCCC-3', Vß12: 5'-CCACTCTGAAGATTCAACCTACAGAACCC-3', Vß13: 5'-CAAGATCCAGTCTGCAAAGCAGGG-3', Vß14: 5'-GCACGGAGAAGCTGCTTCTCAGCC-3', Vß15: 5'-GCATATCTTGAAGACAGAGGC-3',

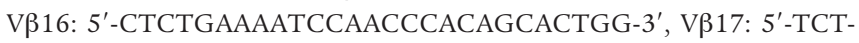
GAAGAAGACGACTCAGCACTG-3', Vß18: 5'-GCAAGGCCTGGAGACAGCAGTATC- $3^{\prime}$ ) and constant region of TCR $\beta$-chain (C $\left.\beta\right)$ : $5^{\prime}$-GCAAGGCCTGGAGACAGCAGTATC-3' $(44,45)$. The amplification was performed in an Eppendorf thermocycler (Mastercycler; Eppendorf) starting with a 2-minute $94^{\circ} \mathrm{C}$ denaturation, followed by 30 cycles consisting of 20 seconds at $94^{\circ} \mathrm{C}, 12$ seconds at $55^{\circ} \mathrm{C}$, and 30 seconds at $68^{\circ} \mathrm{C}$ and a final elongation step of 10 minutes at $68^{\circ} \mathrm{C}$. V $\beta 5.1, \mathrm{~V} \beta 12$, and $\mathrm{V} \beta 16 \mathrm{PCR}$ products were subcloned and sequenced across the CDR3 region as described previously (3). Sequencing data are provided in Supplemental Tables 1-21.

Sequencing of epitope variant. LCMV plaques were excised and used to infect MC57G monolayers in 12-well plates. After 48 hours RNA was isolated, reverse transcription performed using a poly-T primer, and the $\mathrm{NP}_{205}$ region of the cDNA was PCR amplified using $0.3 \mu \mathrm{M}$ each of flanking primers 5'-GGTCCTCGCTGTTGCTTGGCTTGA-3' and 5'-TGGGGAGGCTCAGTGCAGAAGAAC- $3^{\prime}$, using platinum $P f x$ polymerase (Invitrogen Corp.). The PCR conditions were: (a) $94^{\circ} \mathrm{C}$ for 2 minutes; (b) $94^{\circ} \mathrm{C}$ for 15 seconds, $58^{\circ} \mathrm{C}$ for 30 seconds, and $72^{\circ} \mathrm{C}$ for 60 seconds (repeated 30 times); (c) $72^{\circ} \mathrm{C}$ for 60 seconds. DNA sequencing was done at the UMMS sequencing facility with custom primers $(5 \mathrm{pmol} / \mu \mathrm{l}) 5^{\prime}$-CACCAAGACTAAAGTTATAGCCAG-3' and 5'-AGGGTGCAAGTGGTGTGGTAAGAG-3'.

Peptide/MHC stabilization assay. TAP-1-deficient RMA-S cells were seeded into 96-well U-bottom plates at $5 \times 10^{5}$ cells per well. Following incubation in a $5 \% \mathrm{CO}_{2}$ incubator at $27^{\circ} \mathrm{C}$ for 4 hours, $\mathrm{NP}_{205}$ peptides were added at various concentrations and incubated overnight. The cells were then stained with $\mathrm{mAb}$ to $\mathrm{H} 2 \mathrm{~K}^{\mathrm{b}}$ (clone AF6 88.5) conjugated with PE and analyzed by FACS.

Statistics. Statistics are expressed as mean \pm SD. Comparisons between groups were performed with the unpaired Student's $t$ test (2-tailed). $P$ values less than 0.05 were considered statistically significant.

Computer modeling. IMMSIM, an agent-based model of the immune system governed by probabilistic events is available at http://www.immsim. org and can be downloaded for research and educational use (46-50). The IMMSIM body consists of epithelial cells in a grid of discrete "interaction sites," where cells of each type (Th1, Th2, B, macrophages) of the immune system are distributed, meet with each other and with antigens, and mount cellular and humoral responses whenever a virus infects and expresses antigens in the target epithelial cells. To simulate the T cell clonal distributions observed in vitro (unique for each individual but partly overlapping), the key decision was to use 16-bit strings to represent the TCR, yielding a theoretical repertoire of 65,536 (216) different receptors and a peptide-specific repertoire of 697.

In the first experiment, we created virtual mice containing 2,500 CD8 T lymphocytes with an average of 27 clones specific for any sorted peptideTCR combination. In the second experiment, in order to analyze a wider clonal distribution, each virtual mouse had 10,000 CD8 T lymphocytes, but the same ratio between the number of specific clones and the individual repertoire was maintained, yielding an average of 108 peptide-specific clones. In both cases the individual repertoires of the mice are different but partly overlapping.

To study the focusing of the memory repertoire as a consequence of $\mathrm{T}$ cell cross-reactivity, we designed 2 different sets of experiments to perform simulations: (a) homologous infection, in which the same virus (with noncross-reacting B epitope) was injected at time steps 0 and 600 , in order to evaluate the effects of a rechallenge on the preexistent memory repertoire; and (b) heterologous infection, in which we performed a heterologous challenge by injecting at time step 0 the same virus used in the previous set and injecting a cross-reacting virus at time step 600 . The 2 cross-reactive viruses were slightly different in their bitstring composition, to simulate the close amino acid sequence relationship between LCMV NP 205 and $\mathrm{PV} \mathrm{NP}_{205}$.

As a measure of focalization, we used the skewness $(S)$ of the CD8 T memory cells population that expresses the degree of asymmetry of the distribution: $S=m_{3} / m_{2}^{3 / 2}$, where $m_{2}$ and $m_{3}$ are the central moments of degrees 2 and 3 , respectively.

To study whether, during a heterologous challenge, affinity can play a significant role in determining the changes of the immune hierarchy, we designed 2 experimental sets: from a pool of 30 different primary responses, we selected 2 cases in which, among the cross-reacting clones against the heterologous peptide, high- and low-affinity cells were present in defined proportions (about $10 \%$ high $/ 90 \%$ low-affinity cells in the first experiment and about $50 \%$ high $/ 50 \%$ low-affinity cells in the second one). The 2 primary responses were elicited against the same virus by using different random seeds to initialize the simulator. The cross-reacting peptides injected dur- 
ing the second challenge are different in their bitstring composition and show a different level of cross-reactivity with the first peptide. A simulated adoptive transfer technique was used to further validate the results by performing different secondary challenges (using diverse random numbers) to stimulate the same memory pool generated in the first response.

\section{Acknowledgments}

We thank Keith Daniels and Frances Saccoccio for technical support and Yuri Naumov, Heiner Wedemeyer, and Eva SzomolanyiTsuda for helpful discussion. This work was supported by NIH research grants AR35506, AI46578, AI054455, and DK52530. Markus Cornberg was supported by a research fellowship (CO

310/1-1) from the Deutsche Forschungsgemeinschaft (DFG). The contents of this publication are solely the responsibility of the authors and do not represent the official view of the NIH.

Received for publication December 29, 2005, and accepted in revised form February 21, 2006.

Address correspondence to: Raymond M. Welsh, Department of Pathology, University of Massachusetts Medical School, 55 Lake Avenue North, Worcester, Massachusetts 01655, USA. Phone: (508) 856-5819; Fax: (508) 856-0019; E-mail: raymond.welsh@ umassmed.edu.

1. Nikolich-Zugich, J., Slifka, M.K., and Messaoudi, I. 2004. The many important facets of T-cell repertoire diversity. Nat. Rev. Immunol. 4:123-132.

2. Davis, M.M., and Bjorkman, P.J. 1988. T-cell antigen receptor genes and T-cell recognition. Nature. 334:395-402.

3. Naumov, Y.N., Hogan, K.T., Naumova, E.N., Pagel, J.T., and Gorski, J. 1998. A class I MHC-restricted recall response to a viral peptide is highly polyclonal despite stringent CDR3 selection: implications for establishing memory T-cell repertoires in "realworld" conditions. J. Immunol. 160:2842-2852.

4. Naumov, Y.N., Naumova, E.N., Hogan, K.T., Selin, L.K., and Gorski, J. 2003. A fractal clonotype distribution in the CD8+ memory T-cell repertoire could optimize potential for immune responses. J. Immunol. 170:3994-4001.

5. Pewe, L.L., Netland, J.M., Heard, S.B., and Perlman, S. 2004. Very diverse CD8 T-cell clonotypic responses after virus infections. J. Immunol. 172:3151-3156.

6. Price, D.A., et al. 2004. T-cell receptor recognition motifs govern immune escape patterns in acute SIV infection. Immunity. 21:793-803.

7. Manfras, B.J., et al. 2004. Oligoclonal CD8+ T-cell expansion in patients with chronic hepatitis $C$ is associated with liver pathology and poor response to interferon-alpha therapy. J. Clin. Immunol. 24:258-271.

8. Meyer-Olson, D., et al. 2004. Limited T-cell receptor diversity of HCV-specific T-cell responses is associated with CTL escape. J. Exp. Med. 200:307-319.

9. Pantaleo, G., et al. 1994. Major expansion of CD8+ Tcells with a predominant $\mathrm{V}$ beta usage during the primary immune response to HIV. Nature. 370:463-467.

10. Wilson, J.D., et al. 1998. Oligoclonal expansions of CD8(+) T-cells in chronic HIV infection are antigen specific. J. Exp. Med. 188:785-790.

11. Borrow, P., et al. 1997. Antiviral pressure exerted by HIV-1-specific cytotoxic T lymphocytes (CTLs) during primary infection demonstrated by rapid selection of CTL escape virus. Nat. Med. 3:205-211.

12. Turner, S.J., et al. 2005. Lack of prominent peptidemajor histocompatibility complex features limits repertoire diversity in virus-specific CD8+ T-cell populations. Nat. Immunol. 6:382-389.

13. McHeyzer-Williams, M.G., and Davis, M.M. 1995. Antigen-specific development of primary and memory T-cells in vivo. Science. 268:106-111.

14. Blattman, J.N., Sourdive, D.J., Murali-Krishna, K., Ahmed, R., and Altman, J.D. 2000. Evolution of the T-cell repertoire during primary, memory, and recall responses to viral infection. J. Immunol. 165:6081-6090.

15. Kedzierska, K., Turner, S.J., and Doherty, P.C. 2004. Conserved T-cell receptor usage in primary and recall responses to an immunodominant influenza virus nucleoprotein epitope. Proc. Natl. Acad. Sci. U. S. A. 101:4942-4947.

16. Lin, M.Y., and Welsh, R.M. 1998. Stability and diversity of T-cell receptor repertoire usage during lymphocytic choriomeningitis virus infection of mice. J. Exp. Med. 188:1993-2005.

17. Sourdive, D.J., et al. 1998. Conserved T-cell receptor repertoire in primary and memory CD8 T-cell responses to an acute viral infection. J. Exp. Med. 188:71-82.

18. Selin, L.K., et al. 2004. CD8 memory T-cells: crossreactivity and heterologous immunity. Semin. Immunol. 16:335-347.

19. Selin, L.K., and Welsh, R.M. 2004. Plasticity of T-cell memory responses to viruses. Immunity. 20:5-16.

20. Welsh, R.M., and Selin, L.K. 2002. No one is naive: the significance of heterologous T-cell immunity. Nat. Rev. Immunol. 2:417-426.

21. Brehm, M.A., et al. 2002. T-cell immunodominance and maintenance of memory regulated by unexpectedly cross-reactive pathogens. Nat. Immunol. 3:627-634.

22. Chen, H.D., et al. 2001. Memory CD8+ T-cells in heterologous antiviral immunity and immunopathology in the lung. Nat. Immunol. 2:1067-1076.

23. Selin, L.K., Varga, S.M., Wong, I.C., and Welsh, R.M. 1998. Protective heterologous antiviral immunity and enhanced immunopathogenesis mediated by memory T-cell populations. J. Exp. Med. 188:1705-1715.

24. Wang, X.Z., et al. 2003. Virus-specific CD8 T-cells in peripheral tissues are more resistant to apoptosis than those in lymphoid organs. Immunity. 18:631-642.

25. Maryanski, J.L., et al. 1996. Single-cell PCR analysis of TCR repertoires selected by antigen in vivo: a high magnitude CD8 response is comprised of very few clones. Immunity. 4:47-55.

26. Turner, S.J., Diaz, G., Cross, R., and Doherty, P.C. 2003. Analysis of clonotype distribution and persistence for an influenza virus-specific CD8+ T-cell response. Immunity. 18:549-559.

27. Trautmann, L., et al. 2005. Selection of T-cell clones expressing high-affinity public TCRs within human cytomegalovirus-specific CD8 T-cell responses. J. Immunol. 175:6123-6132.

28. Yewdell, J.W., and Bennink, J.R. 1999. Immunodominance in major histocompatibility complex class I-restricted T lymphocyte responses. Annu. Rev. Immunol. 17:51-88.

29. Dong, T., et al. 2004. HIV-specific cytotoxic T-cells from long-term survivors select a unique T-cell receptor. J. Exp. Med. 200:1547-1557.

30. Ciurea, A., et al. 2001. CD4+ T-cell-epitope escape mutant virus selected in vivo. Nat. Med. 7:795-800.

31. Lewicki, H., et al. 1995. CTL escape viral variants. I. Generation and molecular characterization. Virology. 210:29-40.

32. Jones, N.A., et al. 2004. Determinants of human immunodeficiency virus type 1 escape from the primary CD8+ cytotoxic T lymphocyte response. J. Exp. Med. 200:1243-1256.

33. Wedemeyer, H., Mizukoshi, E., Davis, A.R., Bennink, J.R., and Rehermann, B. 2001. Cross-reactivity between hepatitis $C$ virus and influenza A virus determinant-specific cytotoxic T-cells. J. Virol. 75:11392-11400.

34. Gruner, N.H., et al. 2000. Association of hepatitis
C virus-specific CD8+ T-cells with viral clearance in acute hepatitis C. J. Infect. Dis. 181:1528-1536.

35. Lechner, F., et al. 2000. Analysis of successful immune responses in persons infected with hepatitis C virus. J. Exp. Med. 191:1499-1512.

36. Lechner, F., et al. 2000. CD8+ T lymphocyte responses are induced during acute hepatitis $\mathrm{C}$ virus infection but are not sustained. Eur. J. Immunol. 30:2479-2487.

37. Chang, K.M., et al. 1997. Immunological significance of cytotoxic T lymphocyte epitope variants in patients chronically infected by the hepatitis C virus. J. Clin. Invest. 100:2376-2385.

38. Urbani, S., et al. 2005. Heterologous T-cell immunity in severe hepatitis C virus infection. J. Exp. Med. 201:675-680.

39. Rehermann, B., and Nascimbeni, M. 2005. Immunology of hepatitis B virus and hepatitis $C$ virus infection. Nat. Rev. Immunol. 5:215-229.

40. Selin, L.K., Nahill, S.R., and Welsh, R.M. 1994. Cross-reactivities in memory cytotoxic $\mathrm{T}$ lymphocyte recognition of heterologous viruses. J. Exp. Med. 179:1933-1943.

41. Van der Most, R.G., et al. 1998. Identification of $\mathrm{Db}$ - and Kb-restricted subdominant cytotoxic T-cell responses in lymphocytic choriomeningitis virus-infected mice. Virology. 240:158-167.

42. Kim, S.K., et al. 2005. Private specificities of CD8 T-cell responses control patterns of heterologous immunity. J. Exp. Med. 201:523-533.

43. Mylin, L.M., et al. 2000. Quantitation of CD8(+) T-lymphocyte responses to multiple epitopes from simian virus 40 (SV40) large T antigen in C57BL/6 mice immunized with SV40, SV40 T-antigentransformed cells, or vaccinia virus recombinants expressing full-length $\mathrm{T}$ antigen or epitope minigenes. J. Virol. 74:6922-6934.

44. Barth, R.K., et al. 1985. The murine T-cell receptor uses a limited repertoire of expressed $V$ beta gene segments. Nature. 316:517-523.

45. Fritz, R.B., Wang, X., and Zhao, M.L. 2000. Alterations in the spinal cord T-cell repertoire during relapsing experimental autoimmune encephalomyelitis. J. Immunol. 164:6662-6668.

46. Behn, U., Celada, F., and Seiden, P.E. 2001. Computer modeling in immunology. In Frontiers of life. A. Lanzavecchia, B. Malissen, and R. Sitia, editors. Academic Press. London, United Kingdom. 611-630.

47. Celada, F., and Seiden, P.E. 1992. A computer model of cellular interactions in the immune system. Immunol. Today. 13:56-62.

48. Seiden, P.E., and Celada, F. 1992. A model for simulating cognate recognition and response in the immune system. J. Theor. Biol. 158:329-357.

49. Celada, F., and Seiden, P.E. 1996. Affinity maturation and hypermutation in a simulation of the humoral immune response. Eur. J. Immunol. 26:1350-1358.

50. Kohler, B., Puzone, R., Seiden, P.E., and Celada, F. 2000. A systematic approach to vaccine complexity using an automaton model of the cellular and humoral immune system. I. Viral characteristics and polarized responses. Vaccine. 19:862-876. 